BMC

Genomics

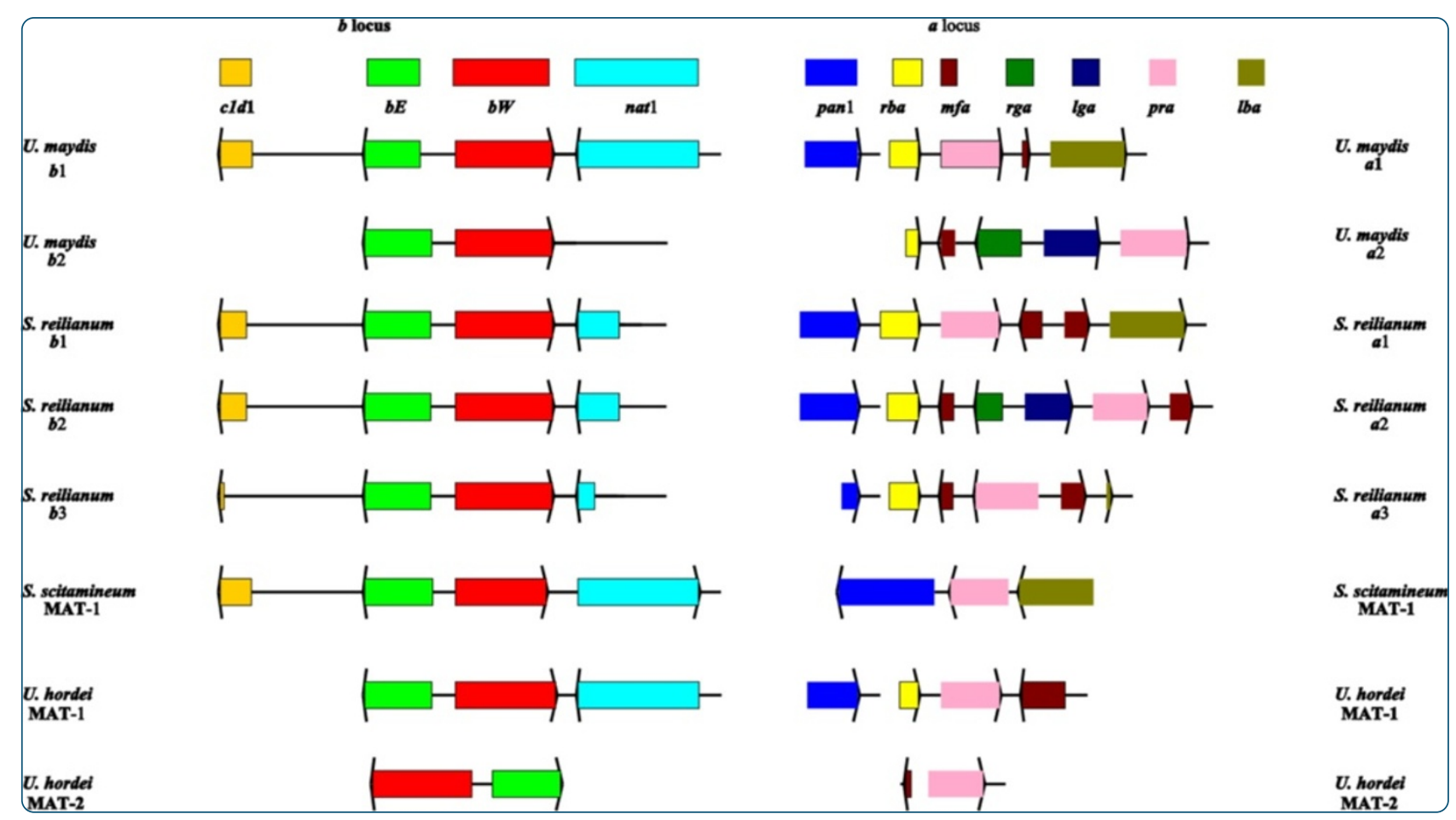

Genome sequencing of Sporisorium scitamineum provides insights into the pathogenic mechanisms of sugarcane smut

Que et al.

() Biomed Central

Que et al. BMC Genomics 2014, 15:996

http://www.biomedcentral.com/1471-2164/15/996 


\title{
Genome sequencing of Sporisorium scitamineum provides insights into the pathogenic mechanisms of sugarcane smut
}

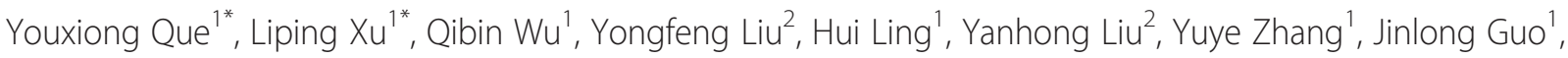
Yachun Su', Jiebo Chen ${ }^{3}$, Shanshan Wang ${ }^{1}$ and Chengguang Zhang ${ }^{2}$

\begin{abstract}
Background: Sugarcane smut can cause losses in cane yield and sugar content that range from 30\% to total crop failure. Losses tend to increase with the passage of years. Sporisorium scitamineum is the fungus that causes sugarcane smut. This fungus has the potential to infect all sugarcane species unless a species is resistant to biotrophic fungal pathogens. However, it remains unclear how the fungus breaks through the cell walls of sugarcane and causes the formation of black or gray whip-like structures on the sugarcane plants.

Results: Here, we report the first high-quality genome sequence of S. scitamineum assembled de novo with a contig $N_{50}$ of $41 \mathrm{~kb}$, a scaffold $\mathrm{N}_{50}$ of $884 \mathrm{~kb}$ and genome size $19.8 \mathrm{Mb}$, containing an estimated 6,636 genes. This phytopathogen can utilize a wide range of carbon and nitrogen sources. A reduced set of genes encoding plant cell wall hydrolytic enzymes leads to its biotrophic lifestyle, in which damage to the host should be minimized. As a bipolar mating fungus, $a$ and $b$ loci are linked and the mating-type locus segregates as a single locus. The S. scitamineum genome has only $6 \mathrm{G}$ protein-coupled receptors (GPCRs) grouped into five classes, which are responsible for transducing extracellular signals into intracellular responses, however, the genome is without any PTH11-like GPCR. There are 192 virulence associated genes in the genome of S. scitamineum, among which 31 expressed in all the stages, which mainly encode for energy metabolism and redox of short-chain compound related enzymes. Sixty-eight candidates for secreted effector proteins (CSEPs) were found in the genome of S. scitamineum, and 32 of them expressed in the different stages of sugarcane infection, which are probably involved in infection and/or triggering defense responses. There are two non-ribosomal peptide synthetase (NRPS) gene clusters that are involved in the generation of ferrichrome and ferrichrome $A$, while the terpenes gene cluster is composed of three unknown function genes and seven biosynthesis related genes.
\end{abstract}

Conclusions: As a destructive pathogen to sugar industry, the S. scitamineum genome will facilitate future research on the genomic basis and the pathogenic mechanisms of sugarcane smut.

Keywords: Sporisorium scitamineum, Sugarcane smut, Pathogenic mechanisms, G-protein coupled receptors, Carbohydrate degrading enzymes, Biotrophic properties, Candidates for secreted effector proteins, Secondary metabolic pathways

\footnotetext{
* Correspondence: qyxmail@126.com; xIpmail@126.com

${ }^{1}$ Key Laboratory of Sugarcane Biology and Genetic Breeding, Ministry of

Agriculture, Fujian Agriculture and Forestry University, Fuzhou 350002, China

Full list of author information is available at the end of the article
} 


\section{Background}

Sugarcane belongs to the Saccharum genus of the Poaceae family (also called Gramineae or true grasses), an economically important seed plant family that includes maize, wheat, rice, sorghum, and many other forage crops. Sugarcane is the world's largest crop. In 2012, the Food and Agriculture Organization estimates that it was cultivated in 101 countries, on approximately 26.1 million hectares, and with a worldwide harvest of 1.83 billion tons. The 10-year trend for sugarcane production has been upward. Sugarcane is not only a cash crop, but it can also be used as livestock fodder. The main product of sugarcane is sucrose, which accounts for $80 \%$ of all sugar produced in the world and $92 \%$ of that in China. It is also used as raw material in human food industries (molasses, rum, bagasse etc.) and is fermented to produce sugarcane ethanol. Sugarcane ethanol represents $40 \%$ of the world's total ethanol fuel.

The increasing world demand for sugar and sugarcane ethanol keeps driving the development of sugarcane agriculture. The average yield of cane stalk is 60-70 tons per hectare per year. However, this figure can vary between 30 and 180 tons per hectare depending on knowledge and crop management approaches. During the production period, a sugarcane crop is sensitive to several biotic and abiotic factors, including 'sugarcane smut' which is a disease caused by the fungus Sporisorium scitamineum. Sugarcane smut has been an increasing large problem in almost all countries where sugarcane is grown. It was first reported in 1877 in the Natal region of South Africa [1]. Sugarcane smut can cause substantial losses in cane yield and sugar content in susceptible varieties. Losses can range from $30 \%$ to total crop failure, and the disease even leads to variety elimination due to susceptibility to this fungus [2].

The smuts are multicellular fungi-in spite of the unicellular yeast phase, which are characterized by their large numbers of dark, thick-walled and dust-like teliospores. Ustilago, Sporisorium and Macalpinomyces are the three genera of smut fungi (subphylum Ustilaginomycotina). These three genera are comprised of approximately 530 described species that all infect grasses [3]. Compared to the ambiguous position of Macalpinomyces, previous studies have demonstrated that Ustilago and Sporisorium together form a monophyletic group with the Ustilaginomycotina $[4,5]$. The close relationship between Ustilago and Sporisorium can also be evidenced by the misclassification of S. scitamineum as U. scitaminea. Generally, Ustilago infects all aerial parts of the plant and rapidly forms galls or tumors filled with spores. In contrast, Sporisorium infects young seedlings, remains asymptomatic, and grows systemically until the emergence of mass of sooty spores.

S. scitamineum, previously known as U. scitaminea, is a basidiomycete and the causal agent of the sugarcane smut disease. It is among the most important phytopathogenic fungi in sugarcane and has significant impact on sugarcane production. This pathogen results in thin stalks, stunted plant growth, and an outgrowth of fungus of the stalk on the cane known as culmicolous [2] (Figure 1). The infected plants have increased tillering that results in more slender and much weaker leaves. The most recognizable characteristic of this disease is a black or gray outgrowth of a whip-like structure that is referred to as a "smut whip" [1] (Figure 1). These structures emerge from the terminal bud or from the lateral shoots of infected stalks and are composed of both plant and fungal tissues (Figure 1). Within these whip-like structures are millions of teliospores that are responsible for the quick dissemination of the disease. Teliospores germinate and undergo meiosis to form four haploid basidiospores. However, haploid cells are not infective and only the dikaryotic hyphae formed by fusion of compatible sporidia can infect the host. It is assumed that developing varieties of sugarcane resistant to sugarcane smut is the only economical method for control of this disease and the best course of action for management. During the selection process of sugarcane breeding, breeders have to abandon the development of a cross combination once smut disease appears in any of the progeny.

Despite the high worldwide losses in cane yield and sucrose caused by S. scitamineum, a limited amount of information is available about its genome structure and pathogenic mechanisms. A better understanding of the mechanisms of smut fungus-sugarcane interactions is necessary for the development and deployment of more effective and durable resistant cultivars. However, sugarcane smut fungi are biotrophic pathogens that cannot be cultured on artificial media. Most of their life stages, including urediniospores that are commonly used for aetiological and evolutionary biology studies, are dikaryotic. Therefore, it is extremely difficult to conduct molecular studies and functional characterizations of genes in sugarcane smut fungus. Besides its biological features, the genome is especially interesting because this is the first fungus important to the sugarcane industry to be completely sequenced. The insights from the three sequenced genomes of the biotrophic fungal plant pathogens, Ustilago maydis [6] and Sporisorium reilianum [7] in maize and Ustilago hordei [8] in barley, have highlighted the power of comparative genomics of closely related species for identification of virulence determinants and will no doubt facilitate the elucidation of the pathogenic mechanisms of sugarcane smut.

In the present study, we investigate the pathogenic mechanisms of sugarcane smut by sequencing, assembling, and annotating the genome of the smut fungus S. scitamineum. A comparative genomics study with the three related fungi $U$. maydis, $S$. reilianum and $U$. hordei 


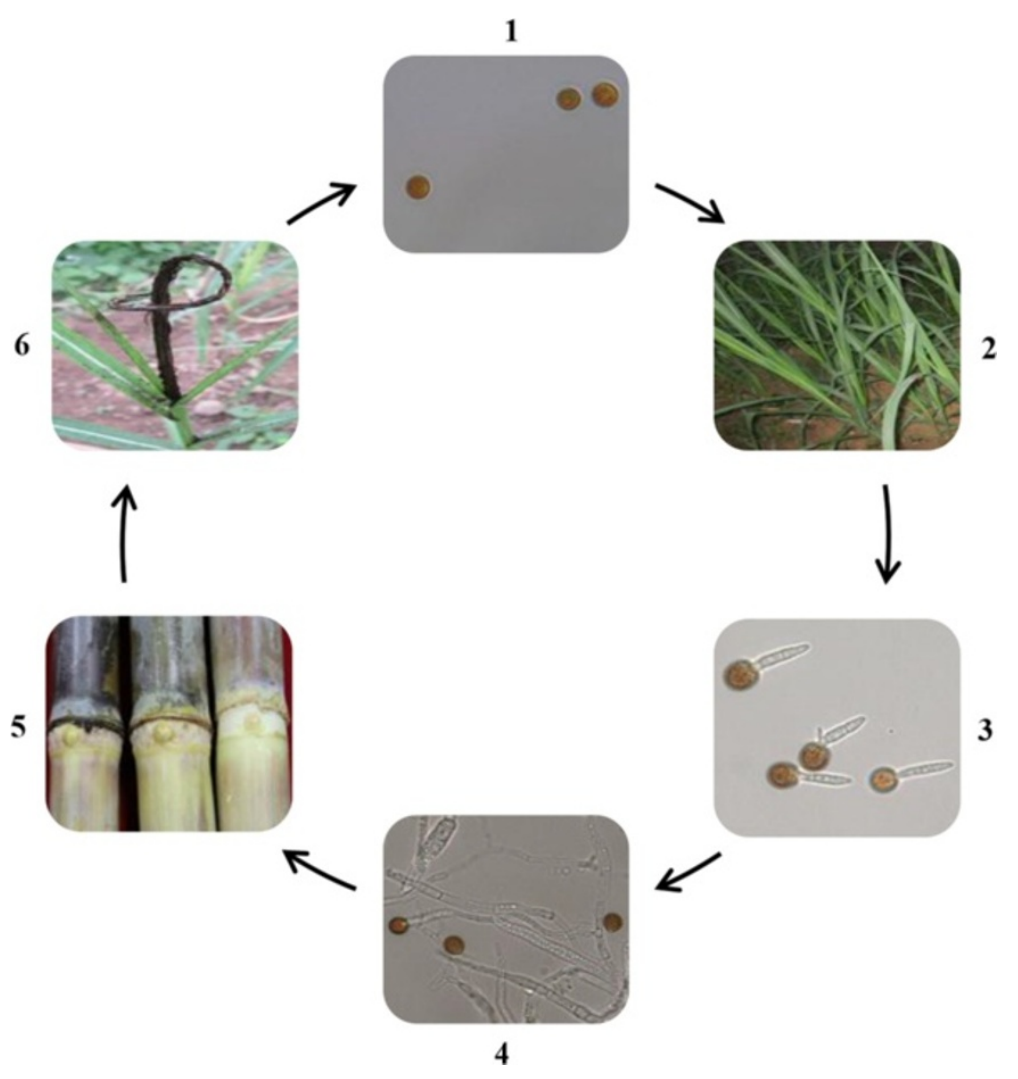

Figure 1 Infection process of Sporisorium scitamineum on sugarcane. 1, Teliospore; 2, Soil or sugarcane plants contaminated with S. scitamineum; 3, Teliospore germination; 4, The formation of infection hyphae; 5, Hypha infection in sugarcane meristem and growth point; 6 , The formation of disease panicle.

was also conducted. This is the first high-quality genome sequence of S. scitamineum and is also the first reported genome of sugarcane fungi. This study may serve as a model for studying the pathogenic mechanisms in sugarcane, and this study provides knowledge for improving the sugar yield in sugarcane agriculture.

\section{Results and discussion}

\section{De novo genome sequencing and assembly}

The genome of $S$. scitamineum was sequenced using a whole-genome shotgun approach. A total of $6.32 \mathrm{~Gb}$ of raw sequences were generated from the Illumina Hiseq2000 platform (Additional file 1: Table S1) at BGI-ShenZhen. The total assembly size of the genome of S. scitamineum is $19.8 \mathrm{Mb}$ which was assembled into 321 contigs and 58 scaffolds (Table 1). According to the k-mer analysis (Additional file 2: Figure S1a), the evaluated genome size of S. scitamineum is $25.9 \mathrm{Mb}$, larger than the assembled one, on account of repeat sequences in the genome. In Additional file 2: Figure S1b, the GC content and depth is normal which illustrates that the genome of $S$. scitamineum has less heterozygosity. The genome properties of S. scitamineum, including genome size, and GC content, is similar to the species closely related to smut fungi, especially $U$. maydis.

\section{Genome annotation}

We predicted 6,636 protein-coding genes with an average transcript size of 1,936 bp via ab initio and homologybased analyses (Additional file 1: Table S2). The gene density was $2.98 \mathrm{~kb}$ per gene, which is lower than that in S. reilianum (2.73 kb per gene) and similar to the other two smut pathogens $U$. maydis (3.01 kb per gene) and $U$. hordei (2.91 kb per gene). The absolute number of exons was largest in S. scitamineum, however, the average exon size was the shortest among these smut fungi. Interestingly, the whole genome character of S. scitamineum is most similar to $U$. maydis which belongs to a separate genus. Only 4,585 (69.09\%), 2,922 (44.03\%), 3,196 (48.16\%), 2,985 (44.98\%), and $6490(97.80 \%)$ of the predicted genes had homologies with known functions in the SwissProt, Gene ontology (GO), Clusters of Orthologous Groups (COG), Kyoto Encyclopaedia of Genes and Genomes (KEGG), and non-redundant proteins (NR) databases, respectively. There were 1,353 genes common to all these classical protein databases (Additional file 1: Table S3).

With regard to non-coding genes, we identified, 150 tRNAs, 5 snRNAs, and 35 rRNA fragments from the assembly (Additional file 1: Table S4). Transposable elements 
Table 1 Sporisorium scitamineum genome statistics compared to that of other sequenced smut fungi

\begin{tabular}{|c|c|c|c|c|}
\hline Genome specifics & Ss & Uh & Um & $S r$ \\
\hline \multicolumn{5}{|l|}{ Assembly statistics } \\
\hline Total contig length (Mb) & 19.8 & 20.7 & 19.7 & 18.2 \\
\hline Total number of contigs & 321 & 2,953 & 275 & 915 \\
\hline Total scaffold length (Mb) & 19.9 & 21.2 & 19.7 & 18.5 \\
\hline Total number of scaffolds & 58 & 713 & 275 & 45 \\
\hline Average base coverage $(X)$ & 213 & 20 & 10 & 20 \\
\hline $\mathrm{N}_{50}$ contig $(\mathrm{kb})$ & 41 & 15.8 & 127 & 36 \\
\hline $\mathrm{N}_{50}$ scaffold $(\mathrm{kb})$ & 884.0 & 307.7 & 127.0 & 772.3 \\
\hline GC content (\%) & 54.85 & 52.00 & 54.00 & 59.70 \\
\hline \multicolumn{5}{|l|}{ Coding sequence } \\
\hline Percentage coding (\%) & 64.4 & 59.9 & 64.1 & 66.9 \\
\hline Average gene size (bp) & 1,936 & 1,782 & 1,935 & 1,852 \\
\hline Average gene density (kb/gene) & 2.98 & 2.91 & 3.01 & 2.73 \\
\hline Protein-coding genes & 6,636 & 7,110 & 6,548 & 6,673 \\
\hline Exons & 11,521 & 10,995 & 11,458 & 9,751 \\
\hline Average exon size (bp) & 1,044 & 1,102 & 1,049 & 1,225 \\
\hline Exons/gene & 1.74 & 1.55 & 1.75 & 1.46 \\
\hline \multicolumn{5}{|l|}{ Noncoding sequence } \\
\hline Introns & 4,885 & 3,161 & 4,920 & 3,078 \\
\hline Introns/gene & 0.74 & 0.44 & 0.75 & 0.46 \\
\hline Average intron length (bp) & 167 & 141 & 128 & 135 \\
\hline NCBI accession & JFOL01000000 & CAGI01000001.1 & NW_101241.1 & FQ311472.1 \\
\hline
\end{tabular}

$\mathrm{N}_{50}$ contig, Size in kilobases of $\geq 50 \%$ of the assembled contigs.

Ss, Sporisorium scitamineum; Uh, Ustilago hordei; Um, Ustilago maydis; Sr, Sporisorium reilianum.

play an important role in fungal pathogens [7]. The S. scitamineum genome is comprised of $1.58 \%$ repetitive elements and DNA transposons are dominant (Additional file 1: Table S5).

\section{Comparative genome analyses}

The homology between S. scitamineum and three other smut fungi was examined via gene families. There were 5,331 gene families shared among all four smut fungi, and there were 48 predicted genes in 21 gene families that appear to be unique to S. scitamineum (Figure 2). Among these unique genes, most have unknown functions. Twenty-seven genes did not receive hits in protein databases, and 11 genes annotated to NR even belong to hypothetical or uncharacterized protein encoding genes. These unique genes could act as a potential mine to explore. Main gene families, which have large gene members, included ATP-binding cassette (ABC) transporter, oligopeptide transporter, P-type ATPase (transport), helicase and integrase (DNA binding), and heat shock protein 70 family.

A phylogenetic analysis revealed the evolutionary relationship among smut fungi and positioning of $S$. scitamineum. It showed that S. scitamineum and S. reilianum are sister taxa and that $U$. maydis branches earlier in the evolutionary history (Figure 3a). The genomes of $U$. maydis [6], S. reilianum [7], and $U$. hordei [8] are all organized in 23 chromosomes. They could be used as references to map the genome of $S$. scitamineum. Our results exhibited a remarkably higher degree of synteny between S. scitamineum and S. reilianum than that between S. scitamineum and $U$. maydis or $U$. hordei (Figure 3b,c,d). Meanwhile, the fact that S. scitamineum and S. reilianum share most common gene families could support the relationship of these two species. We conclude that the chromosome number of S. scitamineum is possibly twenty-three based on the high synteny between $S$. scitamineum and $S$. reilianum and the chromosome numbers of the three smut fungi.

\section{Characterization of mating type loci in S. scitamineum}

For mating to occur, two haploid cells of different mating-type need to recognize each other and fuse to form the infectious dikaryon. Mating is regulated by two loci, $a$ and $b$, which harbor conserved genes. At the $a$ locus, these genes encode pheromones and pheromone 


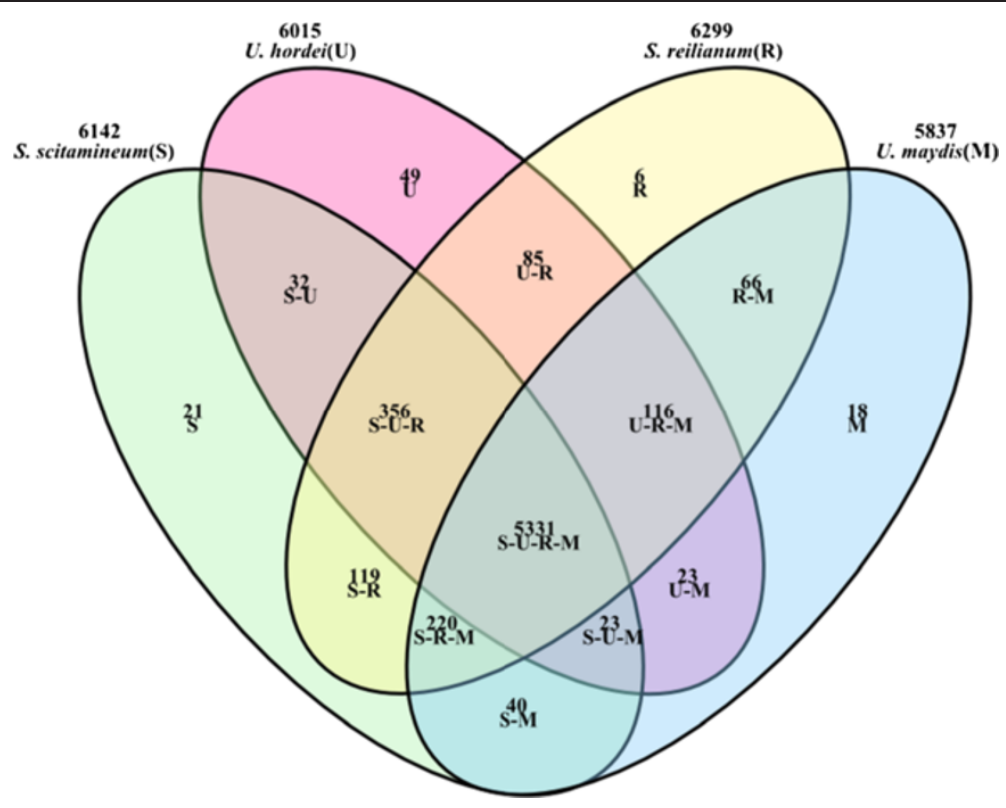

Figure 2 Overlap among gene families for four smut fungi species. This is the venn diagram used to compare the gene families of four smut fungi, four colors (green, red, yellow and blue) mean four gene family sets, and the overlap parts have the overlapping color. Numbers indicate the gene families in each comparison. S means the gene families specific to S. scitamineum, S-U means common gene families of S. scitamineum and U. hordei, S-U-R means common gene families of S. scitamineum, U. hordei and S. reilianum, S-U-R-M means common gene families of four smut fungi.

receptors while at the $b$ locus two subunits of a heterodimeric transcription factor are encoded [9].

The bipolar species S. scitamineum and $U$. hordei as well as the tetrapolar species $U$. maydis and S. reilianum possess one divergently transcribed gene pair that encode the homeodomain proteins bE (HD1) and bW (HD2). The MAT-1 locus, gene order, orientation, as well as the genomic context are conserved in the $b$ mating-type genes except for the $U$. hordei MAT-2 locus (Figure 4). Interestingly, both $b E$ and $b W$ mating-type genes are present in the genomes of Ustilaginaceae including the two genera of Ustilago and Sporisorium.

In addition to the $b$ mating-type complexes, smut fungi contain genes necessary for cell-cell recognition which are located in the $a$ mating type loci. The detailed structure of these loci has been determined for both the MAT-1 alleles of S. scitamineum and $U$. hordei, an allele of $U$. maydis, and for all three alleles of $S$. reilianum (Figure 4). Both $U$. maydis and $U$. hordei have two alleles of an $a$ mating system with one pheromone receptor (pra) and one functional pheromone gene $(m f a)$ per locus.

In S. scitamineum and $U$. hordei which have a bipolar mating system, the $a$ and $b$ loci are linked and the matingtype locus (MAT) segregates as one locus. However, in tetrapolar species such as $S$. reilianum and $U$. maydis, these genetic loci segregate independently [7]. In S. scitamineum, the $a$ locus encodes a lipopeptide with pheromone and pheromone membrane receptor functions responsible for cell recognition and compatible hyphal fusion, whereas the $b$ locus encodes transcription factors that control the expression of genes responsible for the maintenance of the dikaryotic hyphal growth in plants (Figure 4). During their life cycle, S. scitamineum presents two distinct monokaryotic and dikaryotic stages. The monokaryotic stage is marked by haploid cells that grow saprophytically and are not able to cause disease, while in the second phase, dikaryotic hyphae are formed by mating (sexual crossing) and are able to infect the host. The induction of the pathogenic program in S. scitamineum implies not only strong morphological changes (from yeast-like to hyphal) but also genetic changes (haploid to dikaryotic transition).

Evolution of bipolar mating in S. scitamineum may have been beneficial for the fungus because it promoted inbreeding and stabilization of the genome. The same process has been proved to be beneficial for the transposon elements (TEs). A study concluded that inbreeding helped fix TEs within a population in $U$. hordei [10]. In tetrapolar species, such as $U$. maydis and $S$. reilianum, outcrossing increases heterozygosity [7].

Overall, sequence analysis and comparison of the matingtype regions of tetrapolar and bipolar smut fungi revealed that they are not fundamentally different. Bipolar and tetrapolar smuts as well as related species contain the genes for these $a$ and $b$ mating-type complexes. In the bipolar species $S$. scitamineum and $U$. hordei, these mating-type complexes are encoded on the same chromosome and in a recombination-suppressed region ensuring genetic linkage. 
a

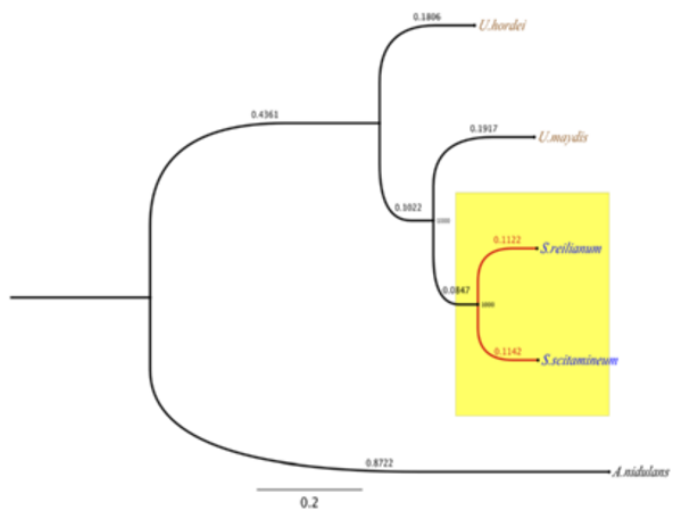

c

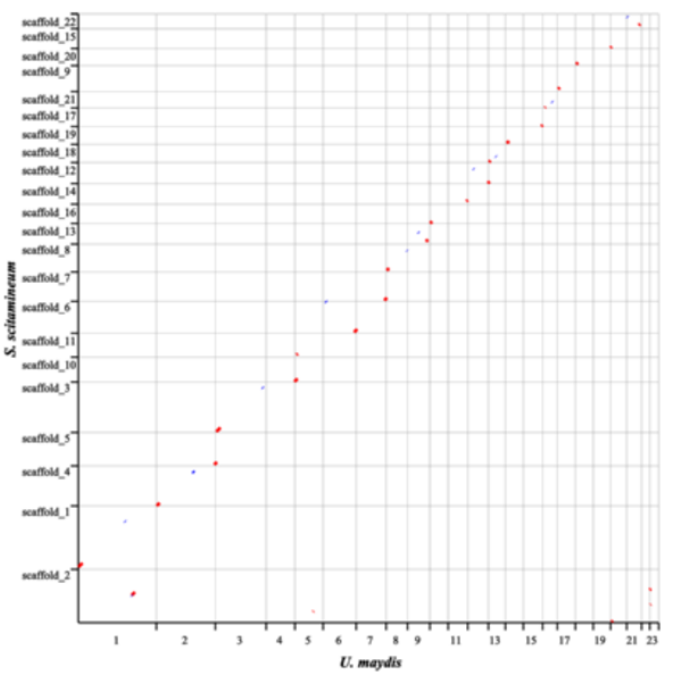

b

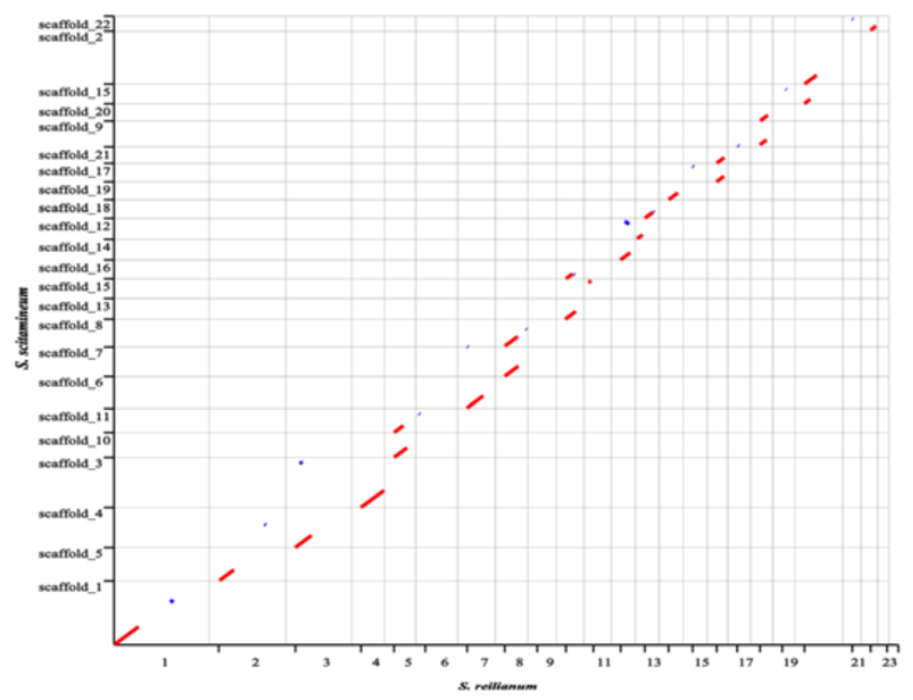

d

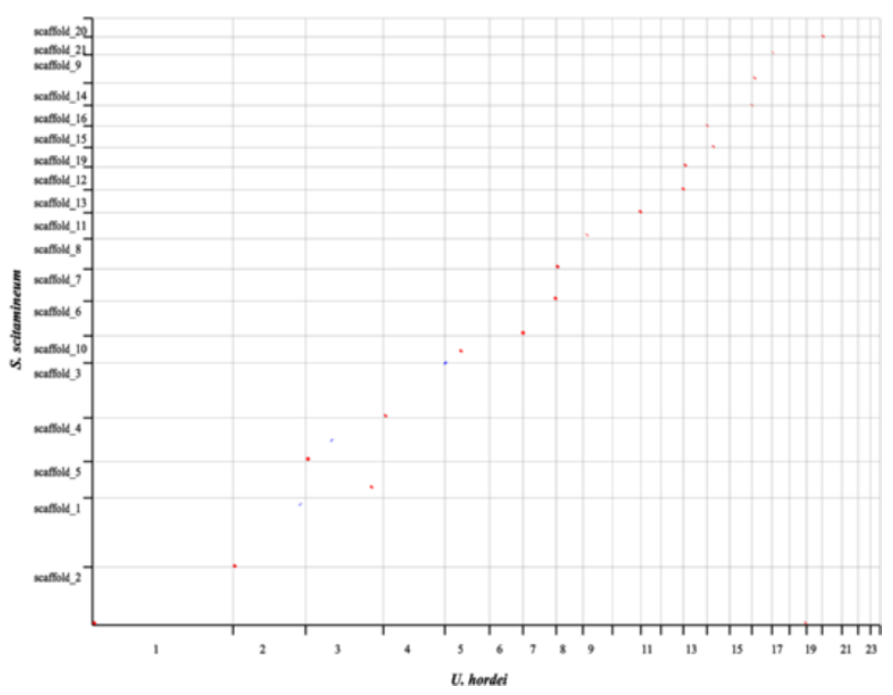

Figure 3 Taxonomic placement of Sporisorium scitamineum and genome synteny with S. reilianum, Ustilago maydis, and U. hordei. (a) Evolutionary placing of S. scitamineum in relation to other smut fungi and A. nidulans. Numbers on the branch indicate branch length. Numbers for the internal node indicate bootstrap value; (b) Dot plot of the synteny occurring between the assembled chromosomes of $S$. reilianum and the assembled scaffolds of S. scitamineum; (c) Dot plot of the synteny occurring between the assembled chromosomes of U. maydis and the assembled scaffolds of S. scitamineum; (d) Dot plot of the synteny occurring between the assembled chromosomes of $U$. hordei and the assembled scaffolds of S. scitamineum.

\section{G-protein-coupled receptors}

To successfully infect a host plant, plant pathogenic fungi and oomycetes must make appropriate responses to a variety of environmental cues, including the chemical and physical characteristics of the host plant surface. This is achieved via cell surface receptors that respond to external stimuli and relay that information into the cell. G protein-coupled receptors (GPCRs), which are characterized by having seven transmembrane helices, represent the largest family of cell surface receptors and are responsible for transducing extracellular signals into intracellular responses that involve complex intracellularsignaling networks [11-14]. The genomes of animals contain large numbers of GPCRs, while the genomes of the model ascomycete yeast species Saccharomyces cerevisiae and Schizosaccharomyces pombe contain only three and four GPCRs [11], respectively. However, a significant number of putative GPCRs have been demonstrated in 


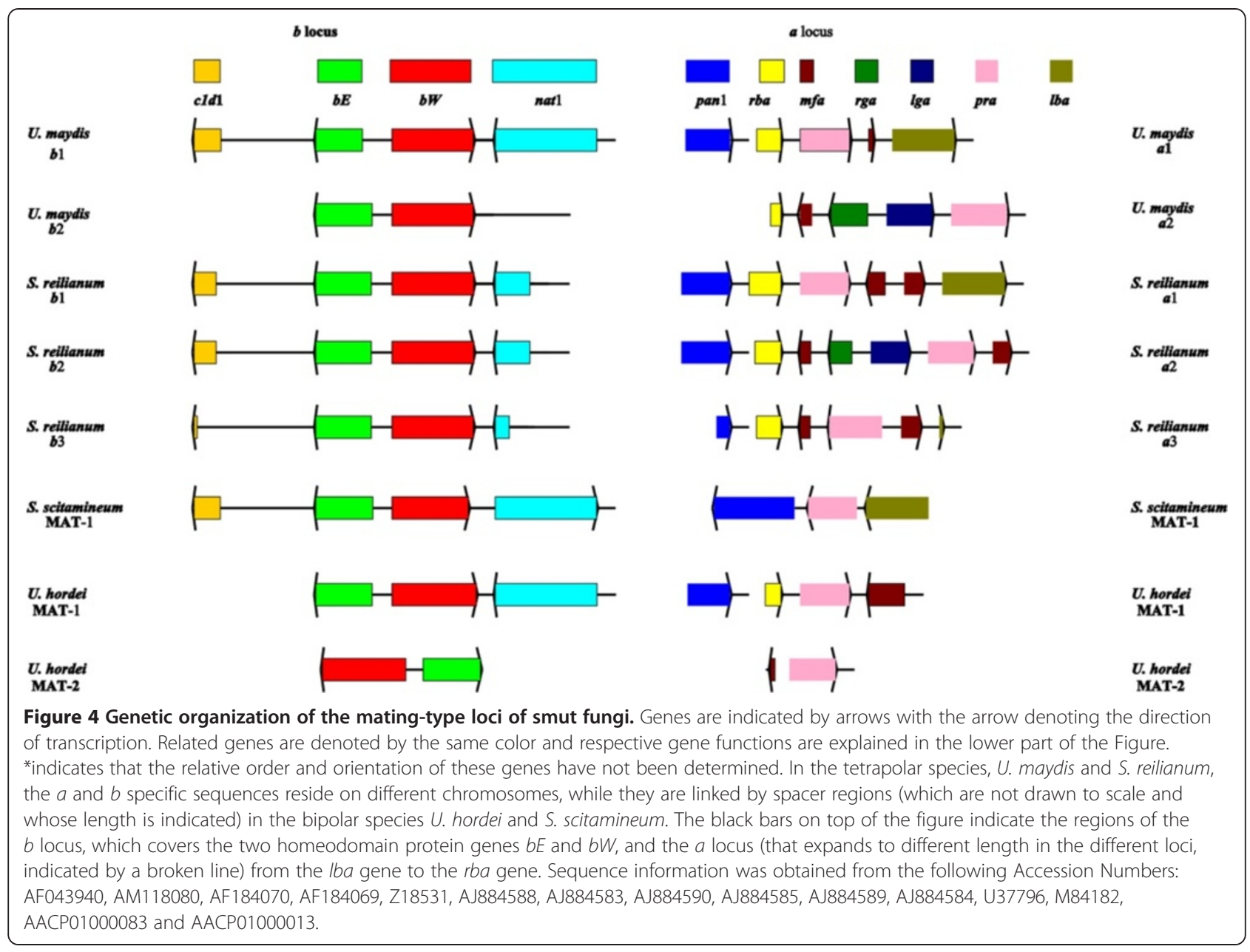

the analysis of the genomes of filamentous phytopathogenic ascomycete fungi, as many as 61 have been reported in the rice blast fungus Magnaporthe grisea and 84 have been demonstrated in the head blight pathogen Fusarium graminearum [12,13]. Previous researches also revealed that GPCRs are extremely diverse in sequence and function. Constraints in structure prediction impaired research on these proteins for a long time [11-16], highlighting the necessity of identifying and characterizing far more GPCRs.

Here we present an exploration of the genomes of 12 fungi, the S. scitamineum plus other 11 fungi, and identify members of the $G$ protein-coupled receptor family from the entire deduced proteomes. It demonstrated that $S$. scitamineum possesses only 6 GPCRs, which are grouped into 5 classes (Table 2). This total set of analyses also resulted in the identification of 7 and 5 putative GPCRs in S. reilianum and $U$. maydis respectively, compared to the 10 predicted GPCRs in the $U$. hordei (Table 2). This suggests that the number of GPCRs in $S$. scitamineum is closer to those of $S$. reilianum and
$U$. maydis than that of $U$. hordei. This is in accordance to the phylogeny of these fungi based on comparative genome analyses showing in Figure 3. PTH11, a cellsurface integral membrane protein required for pathogenicity [14], has seven transmembrane regions and an amino-terminal extracellular cysteine-rich EGF-like domain (CFEM domain). The PTH11-like GPCR is a PHI protein shown to regulate $M$. grisea appressorium differentiation in response to the plant surface [14]. We demonstrated that no PTH11-like GPCRs were found in the genome of all the four sequenced smut fungi, including $S$. scitamineum, $S$. reilianum, $U$. maydis and $U$. hordei (Table 2).

S. scitamineum may acquire the ability to respond to a variety of environmental cues using different GPCRs that activate conserved intracellular signaling pathways and give new triggers to response systems. The identification and characterization of GPCRs will provide insights into how S. scitamineum communicates with its environment and senses the presence of intracellular signaling. 
Table 2 Classification of putative GPCRs identified among various plant pathogen fungi

\begin{tabular}{|c|c|c|c|c|c|c|c|c|c|c|c|c|c|}
\hline \multirow{2}{*}{ GPCR class } & \multicolumn{4}{|c|}{ Biotroph } & \multicolumn{2}{|c|}{ Obligate_biotroph } & \multicolumn{2}{|c|}{ Hemibiotroph } & \multicolumn{4}{|c|}{ Necrotroph } & \multirow{2}{*}{ Characteristics/domains } \\
\hline & Ss & Uh & Um & $S r$ & MIp & Pgt & Mo & $F g$ & $V d$ & Fo & $F v$ & $N h$ & \\
\hline I (pheromone receptors) & 0 & 0 & 0 & 0 & 0 & 0 & 1 & 1 & 1 & 1 & 1 & 1 & STE2-type \\
\hline II (pheromone receptors) & 0 & 1 & 1 & 1 & 4 & 3 & 1 & 1 & 0 & 1 & 1 & 1 & STE3-type \\
\hline $\begin{array}{l}\text { III (related to A. nidulan GprC, GprD, } \\
\text { and GprE) }\end{array}$ & 1 & 1 & 0 & 1 & 3 & 3 & 5 & 2 & 3 & 5 & 4 & 3 & $\begin{array}{l}\text { Git3 (G protein-coupled glucose } \\
\text { receptor) domain }\end{array}$ \\
\hline IV (nitrogen sensors) & 1 & 3 & 1 & 1 & 0 & 2 & 2 & 2 & 2 & 3 & 2 & 2 & PQ-loops \\
\hline V (cAMP receptor-like) & 0 & 0 & 0 & 0 & 0 & 0 & 2 & 4 & 2 & 8 & 5 & 4 & Secretin-family/Dicty_CAR domain \\
\hline VI (GPCRs containing RGS domain) & 0 & 0 & 0 & 0 & 0 & 0 & 2 & 0 & 1 & 2 & 2 & 3 & RGS-domain \\
\hline $\begin{array}{l}\text { VII (related to rat growth hormone } \\
\text { releasing factor) }\end{array}$ & 0 & 0 & 0 & 0 & 0 & 0 & 1 & 0 & 1 & 3 & 2 & 1 & Secretin-like \\
\hline $\begin{array}{l}\text { VIII (related to human steroid } \\
\text { receptor mPR) }\end{array}$ & 0 & 0 & 0 & 0 & 2 & 2 & 3 & 1 & 2 & 2 & 3 & 2 & Hlylll-superfamily \\
\hline IX (microbial opsins) & 2 & 2 & 2 & 2 & 2 & 1 & 1 & 3 & 2 & 6 & 6 & 5 & Bac_rhodopsin \\
\hline X (similar to PTM1) & 1 & 1 & 1 & 1 & 1 & 1 & 1 & 1 & 1 & 1 & 1 & 1 & Lung_7TM superfamily \\
\hline XI (similar to GPCR89) & 0 & 1 & 0 & 0 & 0 & 0 & 0 & 0 & 1 & 0 & 0 & 0 & ABA_GPCR domain \\
\hline XII (family C-like GPCRs) & 0 & 0 & 0 & 0 & 0 & 0 & 2 & 1 & 1 & 3 & 1 & 1 & \\
\hline XIII (related to GPR11 of P. sojae) & 1 & 1 & 0 & 1 & 1 & 5 & 2 & 1 & 0 & 3 & 1 & 1 & DUF300 superfamily \\
\hline PTH11-like & 0 & 0 & 0 & 0 & 0 & 0 & 9 & 2 & 3 & 10 & 5 & 6 & Related to $M$. grisea PTH11 receptor \\
\hline
\end{tabular}

Ss, Sporisorium scitamineum; Uh, Ustilago hordei; Um, Ustilago maydis; Sr, Sporisorium reilianum; Mlp, Melampsora laricis-populina; Pgt, Puccinia graminis f. sp. tritici; Mo, Magnaporthe oryzae; Fg, Fusarium graminearum; Vd, Verticillium dahliae; Fo, Fusarium oxysporum; Fv, Fusarium verticillioides; Nh, Nectria haematococca.

\section{Carbohydrate degrading enzymes}

Phytopathogenic fungi secrete a cocktail of hydrolytic enzymes (including carbohydrateactive enzymes, CAZymes) for degrading the plant cell wall and penetrating into the host tissue. The S. scitamineum genome encoded $867 \mathrm{pu}-$ tative CAZymes including 337 glycoside hydrolases (GH), 253 glycosyltransferases (GT), 92 carbohydrate esterases (CE), 181 carbohydrate binding modules (CBM), and 4 polysaccharide lyases (PL) comprising more than 130 distinct families (Table 3). There were 103 genes that could be annotated by the five databases utilized (SwissProt, GO, KEGG, COG and NR), among which 18 genes are putative plant cell wall hydrolytic enzymes (GH43, GH51, GH78 and PL4 families) [17]. Most of these enzymes belong to GH78 family (13 genes) and are involved in pectin degradation. Interestingly, pectin is not the main part of the sugarcane cell wall [18]. In terms of carbohydrates, the larger amount of hemicelluloses that comprise up to $50 \%$ of sugarcane cell wall [18] could be broken down by the GH43 family (SmutADNA4_GLEAN_10001516, SmutADNA4_GLEAN_10004227) coding xyloglucan:xyloglucosyltransferase and beta;-xylosidase as well as the GH51 family (SmutADNA4_GLEAN_10004371, SmutADNA4 GLEAN_10005166) coding alpha;-L-arabinofuranosidase and alpha-L-arabinofuranosidase. The minimal set of hemicellulases found in S. scitamineum seems perfectly in line with its biotrophic lifestyle, in which damage to the host is minimized and the release of cell wall fragments, which often trigger plant defense responses, is avoided [6].
Real-time quantitative PCR (RT-qPCR) analysis was performed to examine the expression of these 18 genes during the course of infection $(0,12,24,48,96$ and $120 \mathrm{~h}$ postinoculation (hpi)). The result suggests that there are only ten genes of the GH78 family were expressed and half of them expressed in the initial stage of infection (Figure 5

Table 3 Comparative analysis of CAZyme family among various plant pathogen fungi

\begin{tabular}{lcccccccc}
\hline & & CBM & CE & GH & GT & PL & Total & PCW \\
\hline \multirow{4}{*}{ Biotroph } & Ss & 181 & 92 & 337 & 253 & 4 & 867 & 68 \\
& Uh & 178 & 83 & 451 & 276 & 5 & 993 & 122 \\
& Um & 181 & 103 & 346 & 262 & 3 & 895 & 53 \\
Obligate_biotroph & Sr & 183 & 98 & 339 & 248 & 4 & 872 & 54 \\
& Mlp & 287 & 141 & 448 & 292 & 7 & 1,175 & 124 \\
Hemibiotroph & Pgt & 260 & 93 & 415 & 309 & 6 & 1,083 & 85 \\
& Mo & 411 & 177 & 701 & 399 & 9 & 1,697 & 125 \\
& Fg & 373 & 224 & 821 & 439 & 28 & 1,885 & 158 \\
Necrotroph & Vd & 351 & 182 & 757 & 361 & 45 & 1,696 & 202 \\
& Fo & 669 & 421 & 1,587 & 881 & 37 & 3,595 & 351 \\
& Fv & 595 & 331 & 1,294 & 668 & 35 & 2,923 & 238 \\
& Nh & 452 & 334 & 1,230 & 527 & 46 & 2,589 & 254 \\
\hline
\end{tabular}

Ss, Sporisorium scitamineum; Uh, Ustilago hordei; Um, Ustilago maydis; Sr, Sporisorium reilianum; Mlp, Melampsora laricis-populina; Pgt, Puccinia graminis f. sp. tritici; Mo, Magnaporthe oryzae; Fg, Fusarium graminearum; Vd, Verticillium dahliae; Fo, Fusarium oxysporum; Fv, Fusarium verticillioides; Nh, Nectria haematococca. $\mathrm{CBM}$, carbohydrate binding module; $\mathrm{CE}$, carbohydrate esterase; $\mathrm{GH}$, glycoside hydrolases; GT, glycosyltransferase; PL, polysaccharide lyase; PCW, plant cell wall degradation related enzyme. 


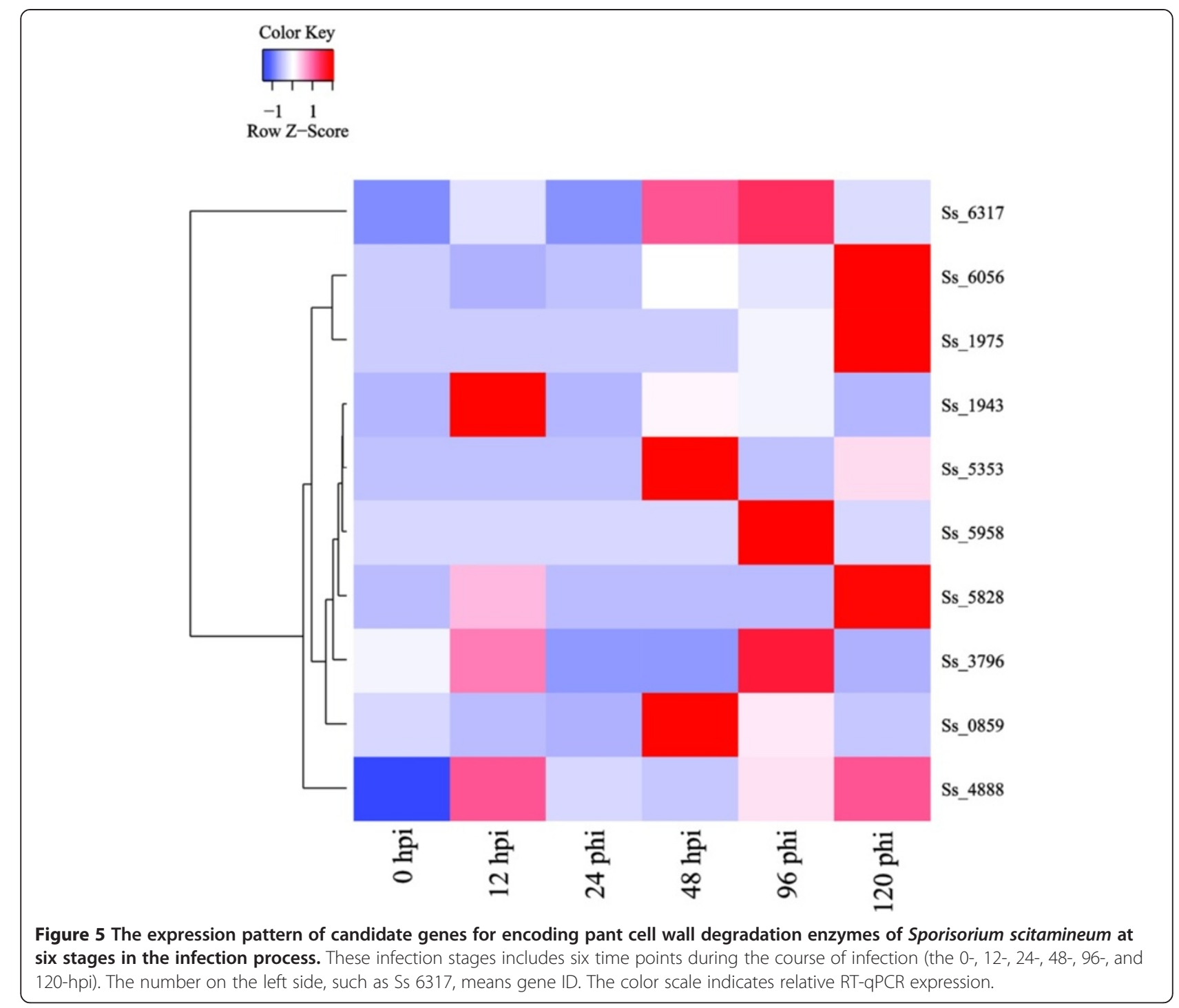

and Additional file 1: Table S6). One gene (SmutADNA4_GLEAN_10004888) encoding alpha;-L-rhamnosidase (EC 3.2.1.40), expressed in all stages and reached the highest expression at $12 \mathrm{hpi}$. It is quite a surprise that no expression was detected for the GH43, GH51 and PL4 families, while the gene expression profiles of the GH78 family are distinct at different infection time points. The regulated expression of ten members of the GH78 family suggests that these key enzymes have a higher activity and play an important role during host infection. However, the expression of a specific gene encoding degradationassociated enzymes should cause damage to the sugarcane plant during infection only at a certain point.

\section{Biotrophic properties and utilization of carbon and nitrogen substrates}

In order to grow and propagate, phytopathogens need to take up nutrients, mainly ammonium and sulfate, from their parasitic host. Obligate biotrophic pathogens which include powdery mildews, rust fungi, downy mildews and Albugo spp., lack genes encoding for particular classes of plant cell wall hydrolases and for various metabolic processes of products, such as nitrates and proteins involved in sulfate assimilation [19]. These losses are interpreted as convergent adaptations.

We anticipated that growth profiles could illuminate correlations between a pathogen and its respective gene complements. Here, we present the comparison of growth on eight carbon and 23 nitrogen substrates. As shown in Figure 6, S. scitamineum can utilize all the eight carbon substrates, which is similar to the assimilation of Cladosporium fulvum and Dothistroma septosporum on carbon substrates [20]. In general, S. scitamineum grows more slowly on the media with carbon substrates of mannitol, lactose, and galactose than on the media with carbon substrates of glucose, fructose, mannose or maltose. The 


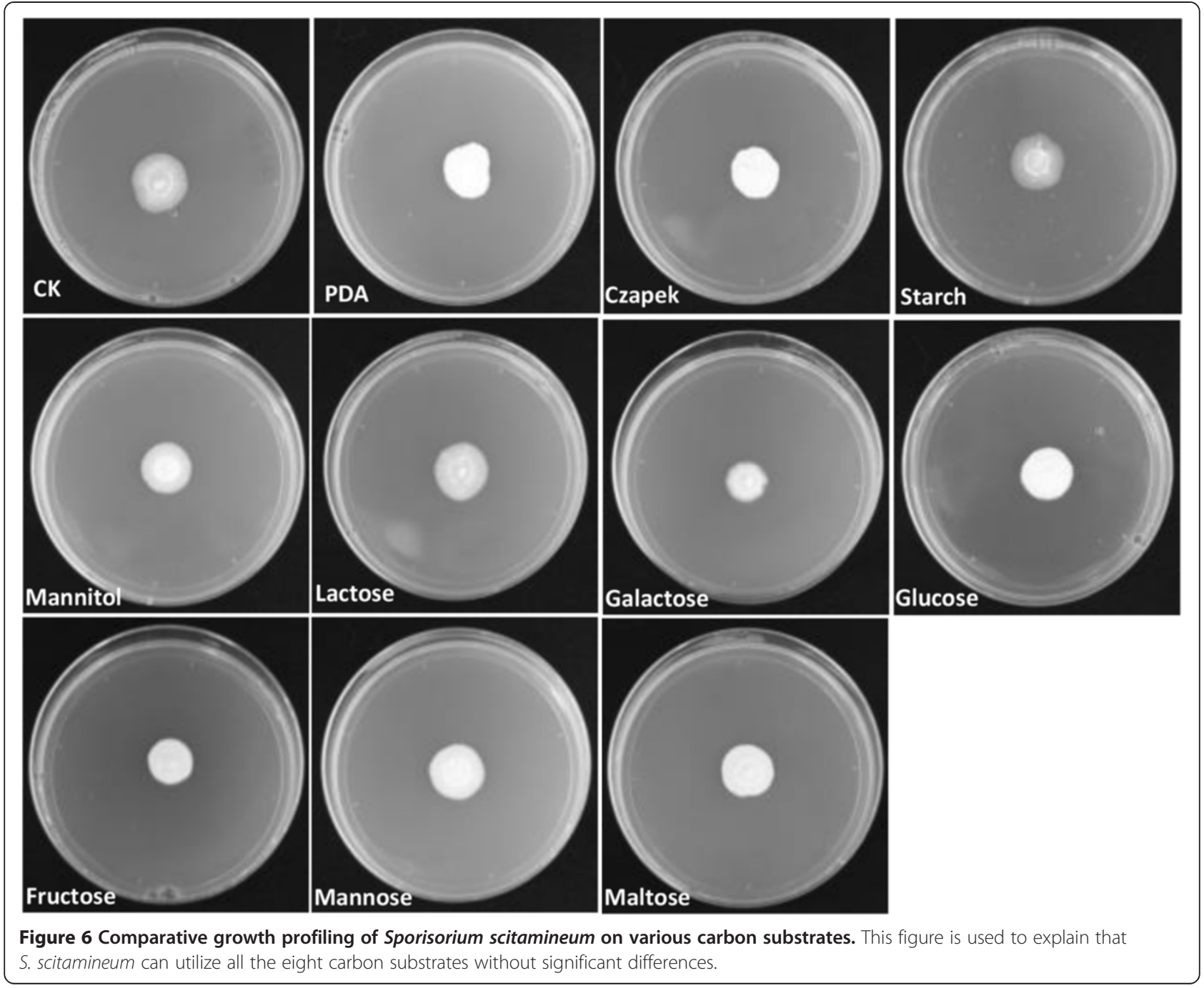

pattern was reversed when the minimal control medium Czapek was also present. The pronounced growth of S. scitamineum on the medium with sucrose is particularly striking and suggests that the fungus can utilize sucrose available in apoplastic fluid during its early biotrophic colonization phase. The result is in accordance with previous reports on C. fulvum [20]. The present study also showed that $S$. scitamineum can uptake a broad range of nitrogen sources, including sodium nitrate, ammonium sulfate, ammonium acetate, and all the twenty kinds of amino acids (Figure 7). The same phenomenon of extensive use of nitrogen sources has also been witnessed in Aspergillus [21] and this emphasizes the impact of nutritional flexibility on fungal pathogenicity. The above-mentioned carbohydrate and nitrogen metabolism pathways including glycolysis, fructose and mannose metabolism, sucrose metabolism, amino acid metabolism, as well as nitrate and sulphate assimilation, are present in the genome of $S$. scitamineum. However, due to the nonuniform growth habit of $S$. scitamineum on these media, further information is needed for conclusions about its ability to utilize carbon and nitrogen substrates. In addition, more experiments must be conducted to support the hypothesis that transporter genes are responsible for the uptake of carbon and nitrogen from plant cells and to confirm the assumption that the broad range of utilization in carbon and nitrogen substrates should result in the wide adaptability of S. scitamineum to various environmental conditions.

The smut fungus $U$. maydis has a reduced set of genes encoding plant cell wall hydrolytic enzymes, presumably as an adaptation to its biotrophic lifestyle [6]. A similar schematic representation of CAZyme is found in the S. scitamineum with the approximate types and sum (Table 3) as typical biotrophic fungi. Biotrophic fungi tend to have fewer plant cell wall degrading enzymes 


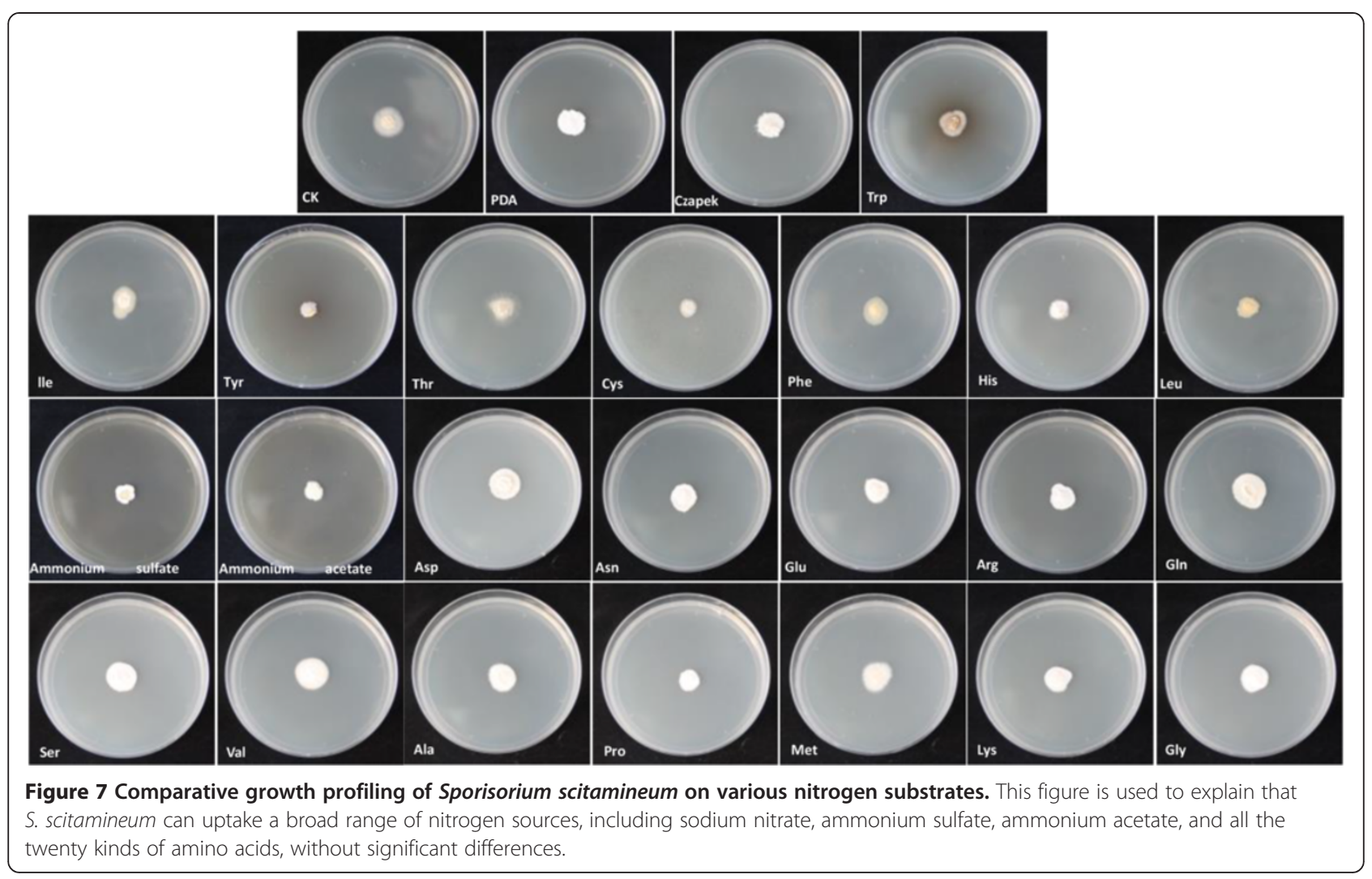

(PCW) than necrotrophic and hemibiotrophic fungi, and the necrotrophic fungi have the most abundant numbers of PCWs.

\section{Virulence associated genes}

As a biotrophic pathogenic fungus, S. scitamineum is expected to possess a number of pathogen-host interaction genes. The genome was searched using the pathogenhost interaction database (PHI-base) [22] and 1,091 putative PHI genes were identified. After filtering the genes from bacteria plant pathogens and animal or human pathogens, there were 192 genes left that could be commonly annotated by five databases (SwissProt, GO, KEGG, COG and NR) as the candidate virulent factor. These 192 genes play diverse roles in redox of shortchain compounds, cell wall breakdown energy metabolism and transport (Additional file 1: Table S7). RT-qPCR analysis was performed to examine the expression profiles of these 192 genes during the course of infection $(0,12,24,48,96$ and $120 \mathrm{hpi})$ and $52 \%$ of them expressed in the process of infection (Figure 8 and Additional file 1: Table S7). The result suggests that 31 genes were expressed in all the stages and mainly encoded energy metabolism and redox of short-chain compound related enzymes (Figure 8 and Additional file 1: Table S7). The gene expression of SmutADNA4_GLEAN_10004570 which encodes peptidyl-prolyl cis-trans isomerase $\mathrm{B}$ (cyclophilin B) continued to increase at all stages and reached its maximum at 120 hpi (a 388 fold change). Cyclophilin B possesses cis-trans peptidyl-prolyl isomerase activity and probably is an important determinant of the virulence of pathogenesis [23]. The function of these virulence associated genes could be elucidated by gene disruption or complementation.

\section{CSEPs and their expression}

Recent researches have revealed the extensive repertoires of pathogen effectors and their various functions. The pathogenicity of S. scitamineum has been studied. However, no effector has been reported prior to our analysis. Effectors have been screened via de novo analysis from the gene set according to the description of Olof Emanuelsson et al. in the Nature PROTOCOL [24]. There are 68 proteins (Figure 9 and Additional file 1: Table S8) that comprise the Candidates for Secreted Effector Proteins (CSEPs) of S. scitamineum which are clustered into 10 families (21 CSEPs) in Table 4. Most of CSEPs in these 10 families have more than $2.0 \%$ cysteine content and longer amino acids. Interestingly, there were just two CSEPs unannotated to the NR database, however, 43 of 66 annotated proteins are conserved hypothetical proteins or uncharacterized proteins. Many of the functions of CSEPs are unknown in biotrophic phytopathogenic fungi, such as $U$. maydis [6] and Blumeria graminis [25]. RT-qPCR 


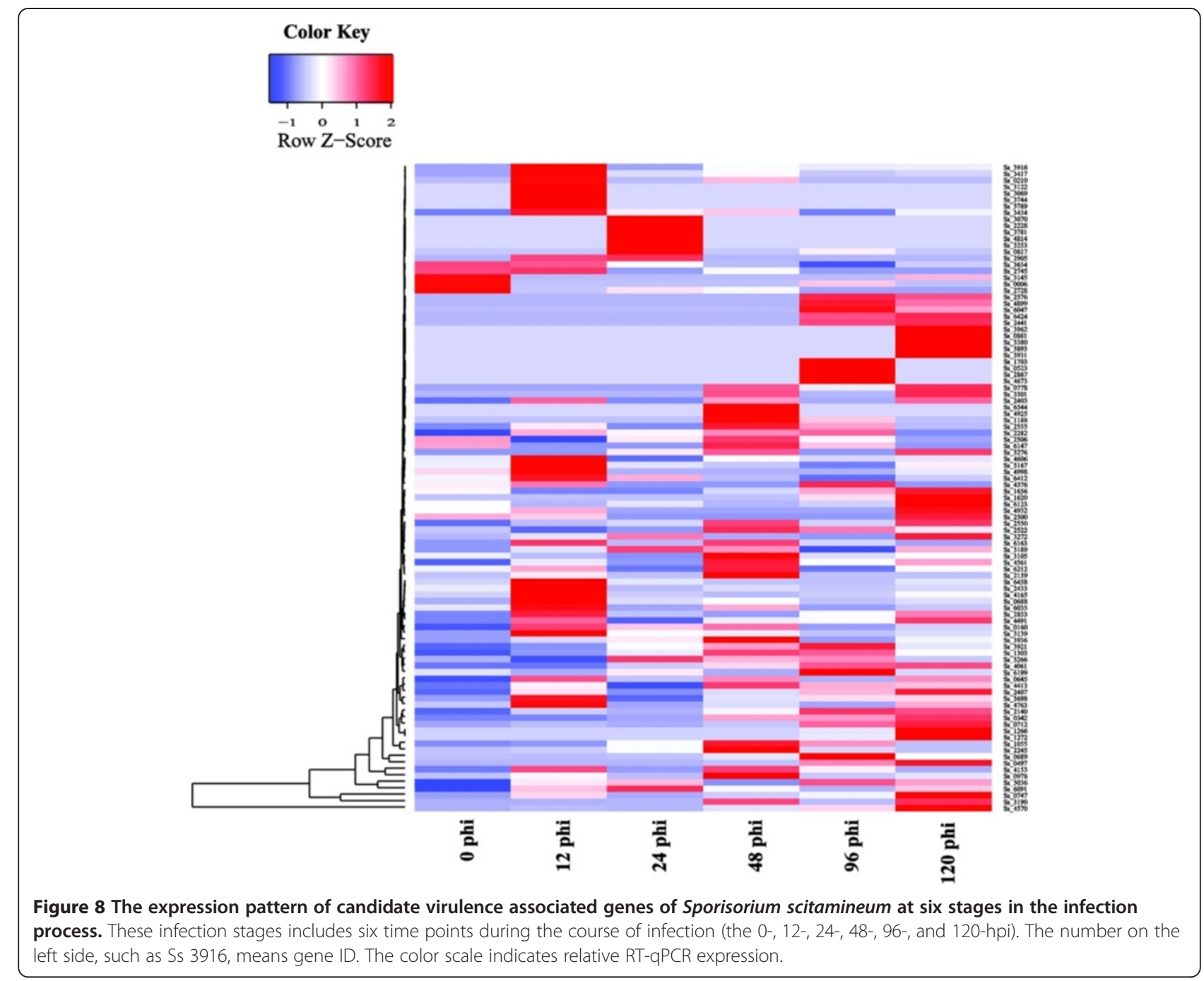

analysis revealed that $47 \%$ of CSEPs expressed in the infection process. Among them, 7 expressed at all stages while $12,9,5,2,2$, and 1 CSEPs started to express at 0, 12, 24, 48, 96, and $120 \mathrm{hpi}$, respectively (Figure 9 and Additional file 1: Table S8). Four of the above-mentioned seven CSEPs belong to conserved hypothetical proteins with unknown function and did not receive hits from any protein database. The remaining three CSEPs have a clan GH-D domain (IPR000111), a catalytic domain (IPR008258), and a Barwin-related endoglucanase domain (IPR009009) as well as sections encoding glycoside hydrolase and lytic transglycosylase-like protein. These CSEPs are mainly involved in carbohydrate degradation. It is unclear how these CSEPs facilitate infection and/or trigger defense responses, how they are secreted and whether there is specific invasion structure to help the secretion. These conundrums require additional endeavors and deeper research.

Protein essential during penetration 1 (Pep1), representing a novel effector for establishing a biotrophic interaction, has been identified and characterized in $U$. maydis [26,27]. Pep1 is also found to be highly conserved in related pathogens such as $S$. reilianum and $U$. hordei $[8,26,27]$. However, all the above 68 CSEPs of S. scitamineum have no ortholog to Pep1. Accordingly, we expanded the search to all proteins and found that one protein encoded by SmutADNA4_GLEAN_10005329, has 176 amino acids with $65 \%$ identity to the $U$. maydis Pep1. It was demonstrated that all Pep1 proteins from the 4 smut fungi have an $\mathrm{N}$-terminal secretion signal and four cysteine residues whose spacing is conserved as well as glycine-rich C-terminal regions (Additional file 2: Figure S2a). In $U$. maydis, a glycine-rich domain of 37 aa at the C-terminus was deleted without affecting biological activity, however, cysteine residues are necessary for secretion of Pep1 [26]. The molecular function of these two regions in S. scitamineum may be similar to that in $U$. maydis. Interestingly, phylogenetic tree based on Pep1 (Additional file 2: Figure S2b) is in accordance with that based on the whole 


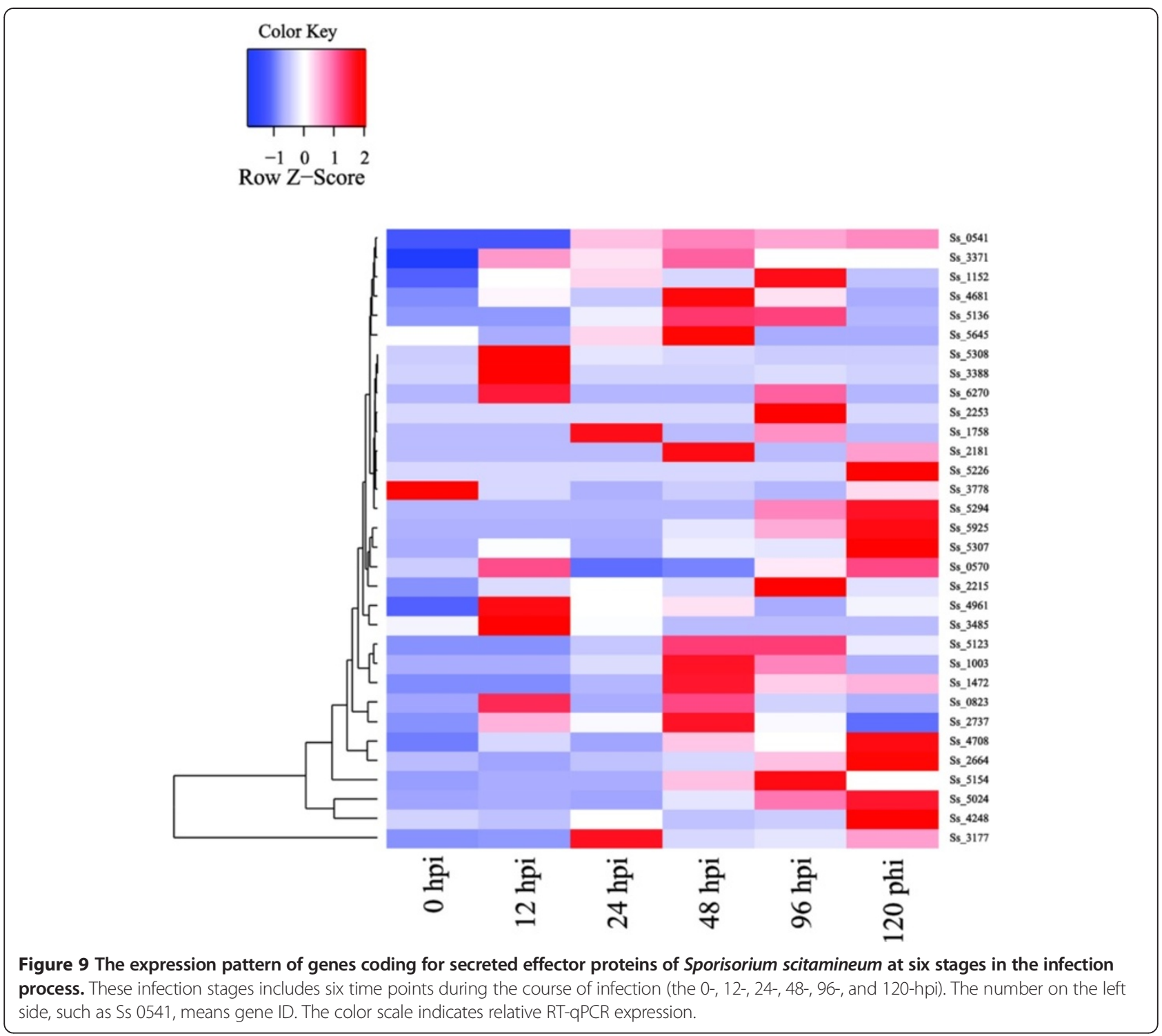

genome (Figure 2), implying that Pep1 can probably be used as a molecular marker for phylogenetic analysis or even for race identification in smut fungi.

\section{Secondary metabolic pathways}

Plant pathogenic fungi produce diverse secondary metabolites that aid in pathogenicity, such as host selective toxins. We identified 145 putative secondary metabolite genes and 11 gene clusters in the S. scitamineum genome. There are 20, 26 and 18 genes predicted to encode polyketide synthases (PKS), non-ribosomal peptide synthetases (NRPS), and terpenes, respectively. Two NRPS gene clusters may participate in iron-chelating siderophores ferrichrome and ferrichrome A biosynthesis. One terpene gene cluster may involve the conversion of gibberellins.

Iron is an important element for many essential processes in living organisms. To acquire iron, S. scitamineum uses two iron-chelating siderophores to uptake iron from host sugarcane cells. In Figure 10a, one NRPS gene cluster including 14 genes, is involved in ferrichrome generation. The core gene SmutADNA4_GLEAN_10002728 encodes ferrichrome siderophore peptide synthetase, and encodes 4,121 amino acids with $76 \%$ identity to the NRPS Sid2 of U. maydis 521 [28]. The gene has three AMP-dependent synthetase/ligase domains, four acyl carrier domains (IPR009081) to activate the cognate amino acid, four condensation domains to catalyze peptide bond formation between the amino acyl and peptidyl substrates of adjacent domains and one AMP-binding enzyme C-terminal domain [29]. The other NRPS gene cluster consists of 12 genes, responsible for ferrichrome A biosynthesis (Figure 10b). The skeleton gene SmutADNA4_GLEAN_10004013 is located in the fifth gene cluster and encodes siderophore peptide synthetase. It encodes 4,590 amino acids with $75 \%$ identity 
Table 4 Summary of the 10 CSEP families in the Sporisorium scitamineum genome

\begin{tabular}{ccccccc}
\hline Family & $\begin{array}{c}\text { Number of } \\
\text { members }\end{array}$ & $\begin{array}{c}\text { C-term } \\
\text { cysteine }\end{array}$ & $\begin{array}{c}\text { Average cysteine } \\
\text { content (\%) }\end{array}$ & $\begin{array}{c}\text { Average peptide } \\
\text { length (aa) }\end{array}$ & IPR annotation & $\begin{array}{l}\text { Expression in the } \\
\text { infection process }\end{array}$ \\
\hline 1 & 3 & Yes & 1.6 & 500 & IPR000560:Histidine phosphatase superfamily, clade-2 & Yes \\
2 & 2 & No & 2.42 & 392 & $\begin{array}{l}\text { IPR013781:Glycoside hydrolase, subgroup, catalytic } \\
\text { domain }\end{array}$ & Yes \\
3 & 2 & Yes & 1.95 & 385 & IPR001283:Allergen V5/Tpx-1-related & Yes \\
4 & 2 & No & 2.03 & 615 & IPR000111:Glycoside hydrolase, clan GH-D & No \\
5 & 2 & No & 2.2 & 567 & IPR001563:Peptidase S10, serine carboxypeptidase & Yes \\
6 & 2 & Yes & 2.61 & 230 & N/A & No \\
7 & 2 & No & 2.05 & 415 & N/A & No \\
8 & 2 & No & 2.12 & 260 & IPR009009:Barwin-related endoglucanase & Yes \\
9 & 2 & No & 2.31 & 346 & N/A & IPR002509:Polysaccharide deacetylase \\
\hline 10 & 2 & Yes & 1.76 & 398 & &
\end{tabular}

to the NRPS Fer3 (CBQ70842.1) of S. reilianum SRZ2, which is composed of three AMP-dependent synthetase/ ligase domains, five acyl carrier domains, four condensation domains and one AMP-binding enzyme C-terminal domain.

Two NRPSs involved in siderophore biosynthesis have also been identified in the ascomycete Cochliobolus heterostrophus and the ferricrocin biosynthesis enzyme is conserved in other ascomycetes [30,31]. A. fumigatus is one of the most common Aspergillus species to cause disease in individuals with an immunodeficiency $A$. fumigatus produces four siderophores, fusarinine $\mathrm{C}$, triacetylfusarinine $\mathrm{C}$, ferricrocin and hydroxyferricrocin [32]. $A$. fumigatus has been recognized as a model species to study fungal siderophore biosynthetic pathways. These two NRPS gene clusters require further study to describe the function of gene members in this cluster by metabolite detection methods such as High Performance Liquid Chromatography (HPLC), Gas Chromatography (GC) and mass spectrometry. Molecular biology experiments also need to be conducted such as knock-out, knock-in, and heterogeneous expression.

In Figure 10c, the terpenes gene cluster is composed of three unknown function genes and seven biosynthesis related genes. The gene SmutADNA4_GLEAN_10000704, which encodes gibberellin 20-oxidase, is known to encode 400 amino acids that have $90 \%$ identity with sr15265 (CBQ69196.1) of S. reilianum SRZ2. Gibberellin 20oxidase is a key oxidase in the biosynthesis of gibberellin (GA). Gibberellin 20-oxidase catalyzes the conversions of GA12 and GA53 to GA9 and GA20 respectively, via a three-step oxidation at C-20 of the GA skeleton. GA35 probably plays an important role in S. reilianum resistance in infected Sorghum bicolor, however, GA20 has no effect [33]. This implies that the gene cluster is involved in weakening the resistance of sugarcane to smut disease and contributes to the successful infection of S. scitamineum.
With GC-MS detection, S. scitamineum can biosynthesize 65 compounds and secrete 13 extracellularly (Additional file 1: Table S9). The intracellular metabolites are mainly esters and the distribution of molecular weight (MW) is from 99 to $475 \mathrm{amu}$. However, the extracellular metabolites are alcohol (2), aldoketones (4), alkanes (2), and carboxylic acids (2) and no esters. The extracellular metabolites exhibited a smaller MW distribution (96-264 amu). Neither intracellular nor extracellular metabolites had strong infection to sugarcane in the inoculation experiments by supernatant (cell-free extracts) and precipitation of fungal elicitor. The questions of whether this was due to the application of mixed metabolites, and if only the single or some secreted metabolites function(s) in the infection process, require further investigation through additional inoculation validation. Determining the function of each metabolite in this process could elucidate the corresponding mechanism causing sugarcane smut.

Cytochrome P450s play an important role in various hydroxylation and oxidation processes including those associated with secondary metabolites as well as the breakdown of toxins and other xenobiotic compounds [34]. Plant pathogenic fungi use a wide range of strategies to gain access to the carbon sources and nitrogen sources of their host plants and to counter the plant defense response. Transporters are involved in toxin and effector secretion as well as nutrition uptake. There are $18 \mathrm{P} 450 \mathrm{~s}$ and 129 transporter genes (Table 5) in the genome of $S$. scitamineum. A large proportion of transporters belong to the major facilitator superfamily (MFS) (74), but the ABC superfamily (17), P-type ATPase (P-ATPase) Superfamily (18), and Sugar/inositol transporter (20) are all well represented. These four smut fungi have similar composition of P450s and transporters. Compared to the typical pathogens M. grisea or Fusarium graminearum [35], the MFS number of S. scitamineum is small. It is unsurprising that few metabolites could be secreted extracellularly. 


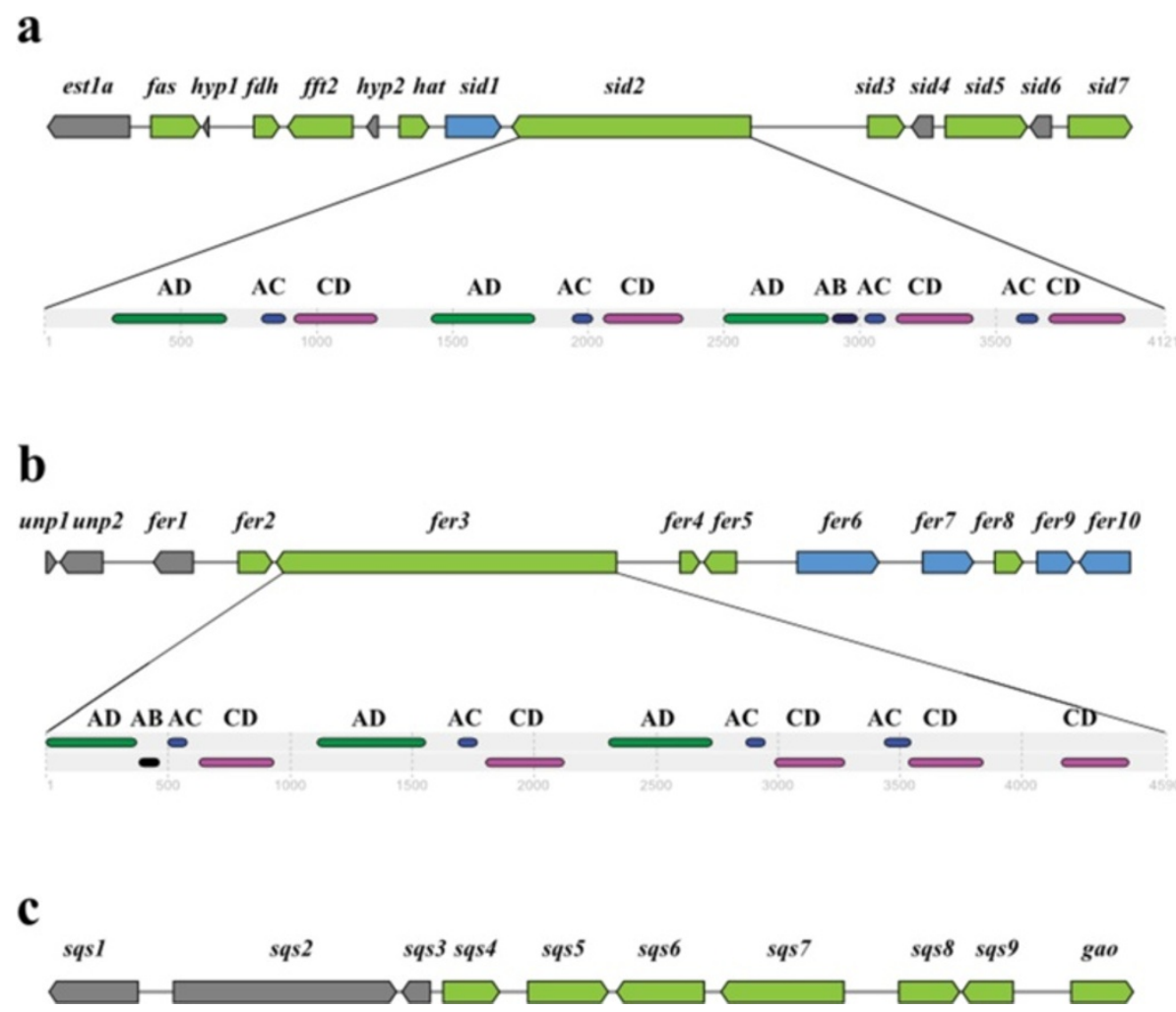

Figure 10 Three gene clusters in the genome of Sporisorium scitamineum. (a) Physical map of ferrichrome gene cluster (SmutADNA4_GLEAN_ 10002720-10002733). Coding regions are indicated with arrows and are putatively involved in ferrichrome biosynthesis (green yellow), siderophore transport (blue), or unknown functions (grey). Location of InterPro domains in NRPS SmutADNA4_GLEAN_10002728 is as bottom. AD, AMP-dependent synthetase/ligase domain (green), IPR00873; AC, acyl carrier domain (purple), IPR009081; CD, condensation domain (red), IPR001242; AB, AMP-binding enzyme C-terminal domain (black), IPR025110. (b) Physical map of ferrichrome A gene cluster (SmutADNA4_GLEAN_10004009-10004020). Coding regions are indicated with arrows and are putatively involved in ferrichrome A biosynthesis (green yellow), siderophore transport (blue), or unknown functions (grey). Location of InterPro domains in NRPS SmutADNA4_GLEAN_10004013 is as bottom. AD, AMP-dependent synthetase/ligase domain (green), IPR00873; AC, acyl carrier domain (purple), IPR009081;CD, condensation domain (red), IPR001242; AB, AMP-binding enzyme C-terminal domain (black), IPR025110. (c) Physical map of terpene gene cluster (SmutADNA4_GLEAN_10000695-10000704). Coding regions are indicated with arrows and are putatively involved in terpene biosynthesis (green yellow) or unknown functions (grey).

\section{Conclusions}

Although sugarcane smut was first reported in 1877, little is known about its pathogenic mechanisms. The S. scitamineum genome sequence and our comparative analysis with the genome sequences of $\mathcal{U}$. maydis, S. reilianum and $U$. hordei have shed new light on the pathogenic mechanisms of this fungus and have provided insights into aspects of genome evolution, biotrophy features, carbohydrate degrading enzymes and secondary metabolic pathways likely to be common to all smut fungi. These results represent the initial step in realizing the full potential of these genomes. As a result of the genome analysis, efforts

Table 5 Comparative analysis of P450s and transporter genes among various plant pathogen fungi

\begin{tabular}{|c|c|c|c|c|c|c|c|c|c|c|c|c|}
\hline \multirow[b]{2}{*}{ Protein families } & \multicolumn{4}{|c|}{ Biotroph } & \multicolumn{2}{|c|}{ Obligate_biotroph } & \multicolumn{2}{|c|}{ Hemibiotroph } & \multicolumn{4}{|c|}{ Necrotroph } \\
\hline & Ss & Uh & Um & Sr & MIp & Pgt & Mo & $F g$ & $V d$ & Fo & $F v$ & Nh \\
\hline Cytochrome P450 & 18 & 25 & 31 & 25 & 33 & 23 & 134 & 126 & 72 & 238 & 148 & 173 \\
\hline Major facilitator superfamily & 74 & 70 & 74 & 74 & 65 & 57 & 181 & 245 & 190 & 179 & 236 & 447 \\
\hline ATP-binding cassette (ABC) superfamily & 17 & 14 & 19 & 16 & 9 & 12 & 27 & 32 & 18 & 27 & 32 & 38 \\
\hline Sugar/inositol transporter & 20 & 18 & 18 & 21 & 19 & 13 & 52 & 87 & 66 & 52 & 115 & 145 \\
\hline P-type ATPase (P-ATPase) superfamily & 18 & 8 & 17 & 18 & 17 & 16 & 12 & 27 & 22 & 23 & 31 & 34 \\
\hline
\end{tabular}

Ss, Sporisorium scitamineum; Uh, Ustilago hordei; Um, Ustilago maydis; Sr, Sporisorium reilianum; Mlp, Melampsora laricis-populina;

Pgt, Puccinia graminis f. sp. tritici; Mo, Magnaporthe oryzae; Fg, Fusarium graminearum; Vd, Verticillium dahliae; Fo, Fusarium oxysporum;

Fv, Fusarium verticillioides; Nh, Nectria haematococca. 
are underway to validate the function of all these potential pathogenicity determinants, especially the virulence associated genes. However, the genome sequence of S. scitamineum provides only a first glimpse into the genomic basis of pathogenic mechanisms of sugarcane smut. These efforts and ongoing sequencing projects for additional sugarcane fungi will provide extraordinary opportunities for comparative analyses that will expand our understanding of the interaction between sugarcane and fungi in this agriculturally and industrially important crop. Further sequencing of S. scitamineum isolates from different geographical locations or different host varieties will lead to comprehensive population genetic analysis.

\section{Methods}

\section{Ethics statement}

No specific permissions are required for these locations/ activities. The field studies do not involve endangered or protected species and are conducted in accordance with local legislation.

\section{Strain, growth condition, and genomic DNA and RNA isolation}

Smut infected whips were collected from sugarcane cultivar "ROC"22 maintained at the Key Laboratory of Sugarcane Biology and Genetic Breeding, Ministry of Agriculture (Fuzhou, China). Teliospores were mixed, sealed in plastic bags and then stored at $4^{\circ} \mathrm{C}$. These samples were subjected to gradient dilution with sterile water and then plated onto potato dextrose agar (PDA) medium which contained $100 \mu \mathrm{g} / \mathrm{mL}$ streptomycin (Sangon Biological Engineering Technology \& Services Co., Ltd, Shanghai, China). Plates were incubated in the dark at $28^{\circ} \mathrm{C}$ for $5 \mathrm{~d}$. Single colonies were transferred onto the new PDA medium and cultured at $28^{\circ} \mathrm{C}$ for $7 \mathrm{~d}$. A single colony named SmutA (haploid) was transferred into potato dextrose water (PDW) medium with $100 \mu \mathrm{g} / \mathrm{mL}$ streptomycin and incubated at $28^{\circ} \mathrm{C}, 200$ rpm for $5 \mathrm{~d}$. DNA from fungal hyphae was extracted using the SDS method and eluted with sterile water containing $100 \mu \mathrm{g} / \mathrm{mL}$ RNase A. The DNA sample was quantified by the NanoVue Plus Spectrophotometer (GE Healthcare, NJ, USA) using the absorbance at $260 \mathrm{~nm}$ and $280 \mathrm{~nm}$. The DNA was submitted to genome sequencing at BGIShenzhen (Shenzhen, China). For fungal elicitor preparation, the SmutA was also transferred into potato dextrose water (PDW) medium with $100 \mu \mathrm{g} / \mathrm{mL}$ streptomycin and incubated at $28^{\circ} \mathrm{C}, 200 \mathrm{rpm}$ for $15 \mathrm{~d}$.

A smut spore suspension containing $5 \times 10^{6}$ spores $/ \mathrm{mL}$ in $0.01 \%(\mathrm{v} / \mathrm{v})$ Tween-20 was needle-inoculated on to buds of sugarcane cultivar "ROC" 22 to create the treatment group. All the inoculated buds were then cultured in an incubator at $28^{\circ} \mathrm{C} \pm 0.5^{\circ} \mathrm{C}$ in the condition of $12 \mathrm{~h}$ light/ $12 \mathrm{~h}$ dark. Five phenotypically normal buds were then collected at each of the time points of $0,12,24,48,96$ and
$120 \mathrm{~h}$. Samples were immediately frozen in liquid nitrogen and stored at $-80^{\circ} \mathrm{C}$. Total RNA of these inoculated buds was isolated using the $\mathrm{TRIzol}^{\oplus}$ reagent according to the manufacturer's instructions (Life Technologies Co. Ltd., CA, USA). Dried RNA samples were dissolved in diethylpyrocarbonate-treated water. RNA quality was assessed on $1.0 \%$ denaturing agarose gels, and the quantity was verified using the NanoVue Plus Spectrophotometer (GE Healthcare, NJ, USA) and the Agilent 2100 Bioanalyzer (Agilent Technologies Co. Ltd., CA, USA). The total RNA of "ROC"22 after pathogen inoculation for 0, 12, 24, 48, 96 and $120 \mathrm{~h}$ were subjected to an analysis of expression pattern of each gene by Real-time quantitative PCR (RT-qPCR).

\section{Genome sequencing and assembly}

From the genomic DNA of S. scitamineum, 170 bp and $500 \mathrm{bp}$ PCR-free as well as $6 \mathrm{~kb}$ and $10 \mathrm{~kb}$ DNA sequencing libraries were constructed. A total of 6,316 Mb was generated by the IlluminaHiseq 2000 at BGI-Shenzhen (Shenzhen, China). To ensure the accuracy of assembly, reads with 20 low-quality ( $\leq \mathrm{Q} 2$ ) bases, $5.5 \% \mathrm{Ns}$, or $15 \mathrm{bp}$ overlap between adapter and duplications were filtered. The short reads from the four libraries were assembled by SOAPdenovo 1.04 [36] with optimal assembly acquired with the key parameter $\mathrm{K}=55$.

\section{Comparative genome analysis}

Gene clustering was conducted with OrthoMCL [37] by setting the main inflation value as 1.5 and other parameters at default setting. Gene clustering was done for four smut fungi including S. scitamineum, U. maydis, U. hordei and S. reilianum for gene family analysis. After clustering, we generated multiple alignments of protein sequences for each gene family using MUSCLE [34] and converted the protein alignments to CDS alignments.

The single-copy core genes of those four fungi and the outgroup species (Aspergillus nidulans) were identified using OrthoMCL [37]. After the core-gene identification, we constructed the multiple alignments with MUSCLE [38]. The subprogram phyml of TreeBeST (http://treesoft. sourceforge.net/treebest.shtml) was used to construct a phylogenetic tree with default parameters and 1,000 bootstrap samples.

\section{Gene prediction and annotation}

To predict genes in the assembled genome, both homology-based and de novo methods were used. For the homology-based prediction, proteins of $U$. maydis strain 521 were mapped onto the assembled genome using Genewise [39]. For de novo prediction, Augustus software program [40] was employed using appropriate parameters. Data from these complementary analyses 
were merged to produce a non-redundant reference gene set using GLEAN (http://glean-gene.sourceforge.net/).

Repeat sequences were identified by Repeat Masker version 3.3.0 with Repbase version 18.05, and the following parameters: --nolow -no_is -norna -s -engine wublast -parallel 1 -lib lib; Repeat Protein Mask was also used with parameters: -noLowSimple, - pvalue $=1 \mathrm{e}-4$ [41]. Non-coding RNA was predicted by rRNAmmer 1.2, tRNAscan-SE 1.23, and Rfam 10.1.

The protein-encoding genes were annotated through BLASTp searches in the SwissProt (2012-06), GO (release: 1.419), COG (release: 20090331), KEGG (release: 59), and NR (2013-09-04) databases, at the threshold of e-value $\leq 1 \times \mathrm{e}^{-5}$. The best hit was filtered using a $50 \%$ identity cut-off value.

To identify proteins involved in carbohydrate metabolism, we used the Carbohydrate Active Enzymes (CAZy) database (http://www.cazy.org/). Pathogenicity and virulence associated genes were identified using the PHI-base database (http://www.phibase.org/). The PHI-base database catalogs experimentally verified pathogenicity, virulence, and effector genes from fungal, oomycete, and bacterial pathogens which infect animal, plant, fungal and insect hosts. Putative secondary metabolites (PKS and NRPS) were identified by using antiSMASH [42]. Cytochrome P450s were identified by the Cytochrome P450 Database (http://drnelson.uthsc.edu/CytochromeP450.html).

\section{Effector protein}

The secreted proteins (effectors) of S. scitamineum were analyzed using several prediction algorithms. TargetP 1.1 (http://www.cbs.dtu.dk/services/TargetP/) was used to predict the cleavage sites of the predicted presequences with the "Perform cleavage site predictions" option. SignalP 4.0 (http://www.cbs.dtu.dk/services/SignalP-4.0/) was used to perform signal peptide cleavage site prediction. Transmembrane helices in the proteins were predicted using TMHMM 2.0 (http://www.cbs.dtu.dk/services/ TMHMM-2.0/). The proteins that contained signal peptide cleavage sites and no transmembrane helices were selected as effectors. The proteins with lengths less than 200 amino acids or Cysteine content less than $1.5 \%$ were removed and the candidate effectors were obtained. CSEP clustering was conducted with OrthoMCL [37] by setting the main inflation value at 1.5 and other parameters at default values. GPCRs sequences were evaluated for seven transmembrane regions by Phobius [43] and TMHMM 2.0 with default settings.

\section{Fungal elicitor preparation and GC-MS detection}

The fungal elicitor includes two parts, that from supernatant (cell-free extracts) and the part from precipitation. For the elicitor preparation from cell-free extracts, $100 \mathrm{ml}$ of the $15 \mathrm{~d}$ culturing liquid medium containing
SmutA was filtered with four pieces of gauze, centrifuged at 3,000 rpm for $20 \mathrm{~min}$, filtered with a $0.22 \mu \mathrm{m}$ filter (Merck-Millipore, Tullagreen, Carrigtwohill, Co. Cork, IRL), and kept at $4^{\circ} \mathrm{C}$ until use [44]. For the elicitor preparation from the precipitation, $100 \mathrm{ml}$ of the $15 \mathrm{~d}$ culturing liquid medium containing SmutA was centrifuged at 5,000 $g$ for $10 \mathrm{~min}$. Mycelia were then harvested, washed in distilled water, dried with filter paper, weighted, and then ground to a fine powder in liquid nitrogen. The powder was suspended with $25 \mathrm{ml}$ of $10 \mathrm{mM}$ Tris- $\mathrm{HCl}(\mathrm{pH} 8.8)$. Following 5,000 $\mathrm{g}$ centrifugation for $10 \mathrm{~min}$ at $4^{\circ} \mathrm{C}, 20 \mathrm{ml}$ of $80 \%(\mathrm{v} / \mathrm{v})$ methanol was used to suspend the pellet, and the mixture was shaken for $4 \mathrm{~h}$ at $38^{\circ} \mathrm{C}$. After a further centrifugation at 5,000 $\mathrm{g}$ for $20 \mathrm{~min}$ at $4^{\circ} \mathrm{C}$, the pellet from the mixture was suspended once with $5 \mathrm{ml}$ methanol and dried under nitrogen air flow. The dried pellet was washed with $10 \mathrm{ml}$ of $10 \mathrm{mM}$ phosphate buffer $(\mathrm{pH}$ 6.8) again, re-suspended with $25 \mathrm{ml}$ of the same buffer and the mixture was autoclaved for $20 \mathrm{~min}$ at $120^{\circ} \mathrm{C}$. Finally, the mixture was centrifuged at $10,000 \mathrm{~g}$ for $20 \mathrm{~min}$ at $4^{\circ} \mathrm{C}$ and the clear supernatant was collected and kept at $4^{\circ} \mathrm{C}$ until use [45].

For detection analysis, the cell-free extracts and the extracts from the precipitation were dried at $-80^{\circ} \mathrm{C}$ with a freeze vacuum drier and then dissolved with $10 \mathrm{ml}$ HPLC/spectro-methanol. These samples were analyzed by a GC-MS analyzer (Agilent 7890A-5975C). The column was a DB-5ms elastic quartz capillary column $(30 \mathrm{~m} \times 250 \mu \mathrm{m} \times 0.25 \mu \mathrm{m})$. The carrier gas was helium at a flow rate of $1 \mathrm{ml} / \mathrm{min}$ and the injection volume was $1 \mu \mathrm{l}$. The column temperature program was $50^{\circ} \mathrm{C}$ for $2 \mathrm{~min}$, which ramped from $50^{\circ} \mathrm{C}$ to $250^{\circ} \mathrm{C}$ at $6^{\circ} \mathrm{C} / \mathrm{min}$, and held at $250^{\circ} \mathrm{C}$ for $150 \mathrm{~min}$. In the mass spectroscopy, the ion source temperature was $230^{\circ} \mathrm{C}$, and the ionization mode was EI $(70 \mathrm{eV})$. The constituents were identified and compared with the NIST08 standard MS database.

\section{Carbon and nitrogen source utilization}

For determining the growth profiles on different carbon and nitrogen substrate, we modified the Czapek for use as basal medium by supplementing 3.0\% agar with $0.2 \%$ $\mathrm{NaNO}_{3}, 0.1 \% \mathrm{KH}_{2} \mathrm{PO}_{4}, 0.05 \% \mathrm{MgSO}_{4} \cdot 7 \mathrm{H}_{2} \mathrm{O}, 0.05 \% \mathrm{KCl}$, $0.01 \% \mathrm{FeSO}_{4} \cdot 7 \mathrm{H}_{2} \mathrm{O}$ and $3.0 \%$ sucrose [46]. The original $\mathrm{pH}$ was adjusted to 6.5 unless otherwise indicated. All the chemicals or reagents were purchased from China National Medicines Corporation Ltd. (Shanghai, China). All the chemicals used in the present study were AR grade or equivalent in purity. Millipore water was used for preparation of all media. For strain growth, fungus cake with $\Phi=5 \mathrm{~mm}$ was inoculated onto the medium flat plate and the culture was incubated at $28^{\circ} \mathrm{C}$ in a thermostat incubator for seven days, and then photographed. PDA was set as the internal control medium. Three replicates were conducted. 
In carbon utilization experiments, the basal medium was supplemented with $90 \mathrm{mM}$ of the carbon source that had equivalent carbon content to that of sucrose. Eight types of carbon substrates, including glucose, maltose, fructose, lactose, soluble starch, D-mannitol, $\mathrm{D}$-mannose, and D-galactose were added separately at a final concentration of $5.0 \mathrm{mg} / \mathrm{mL}$. In nitrogen utilization studies, the basal medium was adjusted with $24 \mathrm{mM}$ of the nitrogen source that had equivalent nitrogen content to that of sodium nitrate. Twenty-two kinds of nitrogen substrate, including ammonium sulphate, ammonium acetate, leucine, tryptophan, isoleucine, threonine, cysteine, phenylalanine, histidine, aspartate, asparagine, glutamine, arginine, glutamate, glycine, lysine, serine, valine, alanine, proline, tyrosine, and ethionine were added separately in the medium at a final concentration of $10.0 \mu \mathrm{M}$.

\section{Gene expression}

The method of RT-qPCR followed the instructions of the SYBR Green Master (ROX) (Roche, China) on a 7500 Real-time PCR system (Applied Biosystems, USA). The GAPDH gene (Forward primer: CACGGCCACTGGA AGCA; Reverse primer: TCCTCAGGGTTCCTGATG $\mathrm{CC})$ was chosen as the internal control of the RT-qPCR. According to the sequence of each target gene of SmutA, a pair of specific primers was designed using the Primer Premier 5.0 software [47]. RT-qPCR was carried out with the FastStart Universal SYBR Green Master (ROX) in a $20 \mu \mathrm{L}$ volume containing $10.0 \mu \mathrm{L}$ FastStart Universal SYBR Green PCR Master (ROX), $0.5 \mu \mathrm{M}$ of each primer, and a $1.0 \mu \mathrm{L}$ template $(5 \times$ diluted cDNA). RT-qPCR with distilled water as the template was performed as a negative control. The RT-qPCR reaction conditions were held at $50^{\circ} \mathrm{C}$ for $2 \mathrm{~min}, 95^{\circ} \mathrm{C}$ for $10 \mathrm{~min}, 40$ cycles of $95^{\circ} \mathrm{C}$ for $15 \mathrm{~s}$, and $60^{\circ} \mathrm{C}$ for $1 \mathrm{~min}$ [47]. When the reaction was complete, the melting curve was analyzed. Each RT-qPCR was repeated three times. The $2^{-\Delta \Delta \mathrm{Ct}}$ method was adopted to analyze the RT-qPCR results $[47,48]$. The primer information of candidate virulent factors is listed in the Additional file 1: Table S10.

\section{Data access}

All data contributing to this genome initiative has been deposited at the NCBI under BioProject PRJNA240344; the genome accession number is [JFOL00000000]. The genome version described in this paper is the first version and the accession number is [JFOL01000000].

\section{Additional files}

Additional file 1: Table S1. Data used for S. scitamineum genome assembly and scaffolding. Table S2. Gene prediction in the S.

scitamineum genome. Table S3. Protein database annotation result for proteins of S. scitamineum. Table S4. Noncoding genes in the S. scitamineum genome. Table S5. Repeat elements in the S. scitamineum genome. Table S6. Expression of candidate genes for encoding pant cell wall degradation enzymes of S. scitamineum at six stages in the infection process. Table S7. Candidate virulence associated genes of $S$. scitamineum compared against pathogen-host interaction (PHI) database and their expression at six stages in the infection process. Table S8. Candidate genes for Secreted Effector Proteins in the S. scitamineum genome (aa length $>200$ aa \& Cys (\%) > 1.5\%) and their expression at six stages in the infection process. Table S9. Sixty-five compounds in the elicitor of S. scitamineum detected by GC-MS. Table S10. Primer information of Real-time quantitative PCR for candidate virulent factors.

Additional file 2: Figure S1. The K-mer distribution and GC-depth analysis. (a) 15-mer depth distribution of $32 \mathrm{X}$ data. Analysis $\mathrm{X}$-coordinate is depth, and Y-coordinate is frequency. (b) GC content and depth correlative analysis. X-coordinate is GC content, and Y-coordinate is average depth. Through calculating GC content and average depth, we can analyze whether GC bias exists. If not seriously biased, this scatter diagram takes on the shape similar to Poisson distribution, that is there will be a peak near the GC content of the genome, and the more deviation from it, the lower the depth is. Figure S2. Pep1 is conserved among Sporisorium scitamineum and other sequenced smut fungi. (a) Sequence alignment of S. scitamineum Pep1 (Ss), S reilianum Pep1 (Sr), U. maydis Pep1 (Um), and U. hordei Pep1 (Uh). Identical amino acids are highlighted in green. Red boxes: conserved cysteine residues; black box: putative N-terminal secretion signal; blue box: poorly conserved glycine-rich C-terminal region. (b) Phylogenetic tree based on Pep1 multiple sequence alignment.

\section{Competing interests}

The authors declare that they have no competing interests.

\section{Authors' contributions}

YXQ and LPX designed, initiated, and supervised the project. YXQ and QBW prepared materials. YXQ, QBW, HL, YYZ, JLG, YCS, and SSW performed experiments and contributed to data analysis and validation. YHL and CGZ participated in the genome analysis. JBC participated in the GC-MS detection. $Y X Q, Y F L$, and LPX participated in the genome analysis and wrote the manuscript. All authors read and approved the final manuscript.

\section{Acknowledgements}

This work was funded by the National Natural Science Foundation of China (31101196 and 31340060), the Program for New Century Excellent Talents in Fujian Province University (JA14095), the earmarked fund from the Modern Agriculture Technology of China (CARS-20) and Research Funds for Distinguished Young Scientists in Fujian Provincial Department of Education (JA13090). We appreciate all ideas and constructive criticism from the reviewers.

\section{Author details}

${ }^{1}$ Key Laboratory of Sugarcane Biology and Genetic Breeding, Ministry of Agriculture, Fujian Agriculture and Forestry University, Fuzhou 350002, China. ${ }^{2}$ BGI-Shenzhen, Main Building 11/F, Beishan Industrial Zone, Yantian District, Shenzhen 518083, China. ${ }^{3}$ College of Life Science, Fujian Agriculture and Forestry University, Fuzhou 350002, China.

Received: 10 May 2014 Accepted: 4 November 2014 Published: 19 November 2014

\section{References}

1. Mundkur B: Taxonomy of the sugar-cane smuts. Kew Bull 1939, 10(4):525-533.

2. Hoy J, Hollier C, Fontenot D, Grelen L: Incidence of sugarcane smut in Louisiana and its effect on yield. Plant Dis 1986, 70(1):59-60.

3. Vánky K, Lutz M: Tubisorus, a new genus of smut fungi (Ustilaginomycetes) for Sporisorium pachycarpum. Mycol Balcanica 2011, 8(2):129-135.

4. Begerow D, Stoll M, Bauer R: A phylogenetic hypothesis of Ustilaginomycotina based on multiple gene analyses and morphological data. Mycologia 2006, 98(6):906-916. 
5. McTaggart A, Shivas R, Geering A, Callaghan B, Vánky K, Scharaschkin T: Soral synapomorphies are significant for the systematics of the Ustilago-Sporisorium-Macalpinomyces complex (Ustilaginaceae). Persoonia 2012, 29:63-67.

6. Kämper J, Kahmann R, Bölker M, Ma L-J, Brefort T, Saville BJ, Banuett F, Kronstad JW, Gold SE, Müller O: Insights from the genome of the biotrophic fungal plant pathogen Ustilago maydis. Nature 2006, 444(7115):97-101.

7. Laurie JD, Ali S, Linning R, Mannhaupt G, Wong P, Güldener U, Münsterkötter M, Moore R, Kahmann R, Bakkeren G: Genome comparison of barley and maize smut fungi reveals targeted loss of RNA silencing components and species-specific presence of transposable elements. Plant Cell 2012, 24(5):1733-1745.

8. Schirawski J, Mannhaupt G, Münch K, Brefort T, Schipper K, Doehlemann G, Di Stasio M, Rössel N, Mendoza-Mendoza A, Pester D: Pathogenicity determinants in smut fungi revealed by genome comparison. Science 2010, 330(6010):1546-1548.

9. Bakkeren G, Kämper J, Schirawski J: Sex in smut fungi: structure, function and evolution of mating-type complexes. Fungal Genet Biol 2008, 45(suppl 1):15-21.

10. Blumenstiel JP: Evolutionary dynamics of transposable elements in a small RNA world. Trends Genet 2011, 27(1):23-31.

11. Soanes DM, Richards TA, Talbot NJ: Insights from sequencing fungal and oomycete genomes: what can we learn about plant disease and the evolution of pathogenicity? Plant Cell 2007, 19(11):3318-3326.

12. Dean RA, Talbot NJ, Ebbole DJ, Farman ML, Mitchell TK, Orbach MJ, Thon M, Kulkarni R, Xu JR, Pan $\mathrm{H}$ : The genome sequence of the rice blast fungus Magnaporthe grisea. Nature 2005, 434(7036):980-986.

13. Cuomo CA, Güldener U, Xu JR, Trail F, Turgeon BG, Di Pietro A, Walton JD, Ma LJ, Baker SE, Rep M: The Fusarium graminearum genome reveals a link between localized polymorphism and pathogen specialization. Science 2007, 317(5843):1400-1402

14. Kulkarni RD, Thon MR, Pan H, Dean RA: Novel G-protein-coupled receptor-like proteins in the plant pathogenic fungus Magnaporthe grisea. Genome Biol 2005, 6(3):R24.

15. Gruber S, Omann M, Zeilinger S: Comparative analysis of the repertoire of $G$ protein-coupled receptors of three species of the fungal genus Trichoderma. BMC Microbiol 2013, 13:108.

16. Li L, Wright SJ, Krystofova S, Park G, Borkovich KA: Heterotrimeric G protein signaling in filamentous fungi. Annu Rev Microbiol 2007, 61:423-452.

17. Zhao Z, Liu H, Wang C, Xu JR: Comparative analysis of fungal genomes reveals different plant cell wall degrading capacity in fungi. BMC Genomics 2013, 14(1):274

18. de Souza AP, Leite DC, Pattathil S, Hahn MG, Buckeridge MS: Composition and structure of sugarcane cell wall polysaccharides: implications for secondgeneration bioethanol production. BioEnergy Res 2013, 6(2):564-579.

19. Raffaele $S$, Kamoun S: Genome evolution in filamentous plant pathogens: why bigger can be better. Nat Rev Microbiol 2012, 10(6):417-430.

20. de Wit PJ, Van Der Burgt A, Ökmen B, Stergiopoulos I, Abd-Elsalam KA, Aerts $A L$, Bahkali AH, Beenen HG, Chettri P, Cox MP: The genomes of the fungal plant pathogens Cladosporium fulvum and Dothistroma septosporum reveal adaptation to different hosts and lifestyles but also signatures of common ancestry. PLoS Genet 2012, 8(11):e1003088.

21. Krappmann S, Braus G: Nitrogen metabolism of Aspergillus and its role in pathogenicity. Med Mycol 2005, 43(suppl 1):31-40.

22. Winnenburg $R$, Urban M, Beacham A, Baldwin TK, Holland S, Lindeberg M, Hansen H, Rawlings C, Hammond-Kosack KE, Köhler J: PHI-base update: additions to the pathogen-host interaction database. Nucleic Acids Res 2008, 36(suppl 1):572-576.

23. Viaud MC, Balhadère PV, Talbot NJ: A Magnaporthe grisea cyclophilin acts as a virulence determinant during plant infection. Plant Cell 2002, 14(4):917-930

24. Emanuelsson O, Brunak S, von Heijne G, Nielsen $\mathrm{H}$ : Locating proteins in the cell using TargetP, SignalP and related tools. Nat Protoc 2007, 2(4):953-971.

25. Pedersen C, van Themaat EVL, McGuffin $\sqcup$, Abbott JC, Burgis TA, Barton G, Bindschedler LV, Lu X, Maekawa T, Weßling R: Structure and evolution of barley powdery mildew effector candidates. BMC Genomics 2012, 13(1):694

26. Doehlemann $G$, van der Linde K, Aßmann D, Schwammbach D, Hof A, Mohanty A, Jackson D, Kahmann R: Pep1, a secreted effector protein of Ustilago maydis, is qequired for successful invasion of plant cells. PLOS Pathog 2009, 5(2):e1000290.
27. Hemetsberger C, Herrberger C, Zechmann B, Hillmer M, Doehlemann G: The Ustilago maydis effector Pep1 suppresses plant immunity by inhibition of host peroxidase activity. PLoS Pathog 2012, 8(5):e1002684.

28. Schirawski J: Elucidation of the complete ferrichrome A biosynthetic pathway in Ustilago maydis. Mol Microbiol 2010, 75(5):1260-1271.

29. Finking R, Marahiel MA: Biosynthesis of nonribosomal peptides 1 . Annu Rev Microbiol 2004, 58:453-488.

30. Oide S, Moeder W, Krasnoff S, Gibson D, Haas H, Yoshioka K, Turgeon BG: NPS6, encoding a nonribosomal peptide synthetase involved in siderophoremediated iron metabolism, is a conserved virulence determinant of plant pathogenic ascomycetes. Plant Cell 2006, 18(10):2836-2853.

31. Oide S, Krasnoff SB, Gibson DM, Turgeon BG: Intracellular siderophores are essential for ascomycete sexual development in heterothallic Cochliobolus heterostrophus and homothallic Gibberella zeae. Eukaryot Cell 2007, 6(8):1339-1353.

32. Schrettl M, Bignell E, Kragl C, Sabiha Y, Loss O, Eisendle M, Wallner A, Arst HN $\mathrm{Jr}$, Haynes K, Haas H: Distinct roles for intra-and extracellular siderophores during Aspergillus fumigatus infection. PLoS Pathog 2007, 3(9):e128.

33. Matheussen AM, Morgan PW, Frederiksen RA: Implication of gibberellins in head smut (Sporisorium reilianum) of Sorghum bicolor. Plant Physiol 1991, 96(2):537-544

34. Nelson DR: Cytochrome P450 and the individuality of species. Arch Biochem Biophys 1999, 369(1):1-10.

35. Islam MS, Haque MS, Islam MM, Emdad EM, Halim A, Hossen QMM, Hossain MZ, Ahmed B, Rahim S, Rahman MS: Tools to kill: genome of one of the most destructive plant pathogenic fungi Macrophomina phaseolina. BMC Genomics 2012, 13(1):493.

36. Li R, Zhu H, Ruan J, Qian W, Fang X, Shi Z, Li Y, Li S, Shan G, Kristiansen K: De novo assembly of human genomes with massively parallel short read sequencing. Genome Res 2010, 20(2):265-272.

37. Li L, Stoeckert CJ, Roos DS: OrthoMCL: identification of ortholog groups for eukaryotic genomes. Genome Res 2003, 13(9):2178-2189.

38. Edgar RC: MUSCLE: multiple sequence alignment with high accuracy and high throughput. Nucleic Acids Res 2004, 32(5):1792-1797.

39. Birney E, Durbin R: Using GeneWise in the Drosophila annotation experiment. Genome Res 2000, 10(4):547-548.

40. Stanke M, Keller O, Gunduz I, Hayes A, Waack S, Morgenstern B: AUGUSTUS: $a b$ initio prediction of alternative transcripts. Nucleic Acids Res 2006, 34(suppl 2):435-439

41. Saha S, Bridges S, Magbanua ZV, Peterson DG: Empirical comparison of ab initio repeat finding programs. Nucleic Acids Res 2008, 36(7):2284-2294

42. Medema MH, Blin K, Cimermancic $P$, de Jager $V$, Zakrzewski $P$, Fischbach MA, Weber T, Takano E, Breitling R: antiSMASH: rapid identification, annotation and analysis of secondary metabolite biosynthesis gene clusters in bacterial and fungal genome sequences. Nucleic Acids Res 2011, 39(suppl 2):339-346.

43. Kall $L$, Krogh A, Sonnhammer ELL: A combined transmembrane topology and signal peptide prediction method. J Mol Biol 2004, 338(5):1027-1036.

44. Bruce SA, Saville BJ, Emery RN: Ustilago maydis produces cytokinins and abscisic acid for potential regulation of tumor formation in maize. J Plant Growth Regul 2011, 30(1):51-63

45. Santiago R, de Armas R, Legaz ME, Vicente C: Separation from Ustilago scitaminea of different elicitors which modify the pattern of phenolic accumulation in sugarcane leaves. J Plant Pathol 2008, 90(1):87-96.

46. Ahmad M, Smith E: Utilization of carbon and nitrogen sources and acid/ alkali production by cowpea rhizobia. Plant Soil 1985, 86(2):279-282.

47. Que YX, Su YC, Guo JL, Wu QB, Xu LP: A global view of transcriptome dynamics during Sporisorium scitamineum challenge in sugarcane by RNA-Seq. PLoS One 2014, 9(8):e106476.

48. Livak KJ, Schmittgen TD: Analysis of relative gene expression data using real-time quantitative PCR and the $2^{-\triangle \Delta C T}$ method. Methods 2001, 25(4):402-408.

doi:10.1186/1471-2164-15-996

Cite this article as: Que et al:: Genome sequencing of Sporisorium scitamineum provides insights into the pathogenic mechanisms of sugarcane smut. BMC Genomics 2014 15:996. 